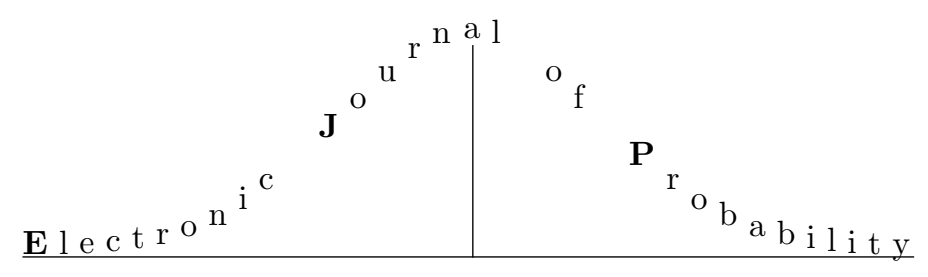

Vol. 13 (2008), Paper no. 10, pages 213-258.

Journal URL

http://www.math. washington.edu/ ejpecp/

\title{
On the approach to equilibrium for a polymer with adsorption and repulsion
}

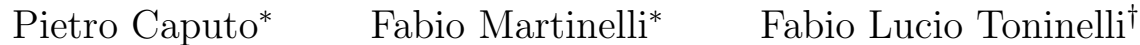

\begin{abstract}
We consider paths of a one-dimensional simple random walk conditioned to come back to the origin after $L$ steps, $L \in 2 \mathbb{N}$. In the pinning model each path $\eta$ has a weight $\lambda^{N(\eta)}$, where $\lambda>0$ and $N(\eta)$ is the number of zeros in $\eta$. When the paths are constrained to be non-negative, the polymer is said to satisfy a hard-wall constraint. Such models are well known to undergo a localization/delocalization transition as the pinning strength $\lambda$ is varied. In this paper we study a natural "spin flip" dynamics for these models and derive several estimates on its spectral gap and mixing time. In particular, for the system with the wall we prove that relaxation to equilibrium is always at least as fast as in the free case (i.e. $\lambda=1$ without the wall), where the gap and the mixing time are known to scale as $L^{-2}$ and $L^{2} \log L$, respectively. This improves considerably over previously known results. For the system without the wall we show that the equilibrium phase transition has a clear dynamical manifestation: for $\lambda \geqslant 1$ relaxation is again at least as fast as the diffusive free case, but in the strictly delocalized phase $(\lambda<1)$ the gap is shown to be $O\left(L^{-5 / 2}\right)$, up to logarithmic corrections. As an application of our bounds, we prove stretched exponential relaxation of local functions in the localized regime.
\end{abstract}

Key words: Pinning model, Spectral gap, Mixing time, Coupling, Dynamical phase transition.

AMS 2000 Subject Classification: Primary 60K35, 82C20.

Submitted to EJP on September 25, 2007, final version accepted February 7, 2008.

\footnotetext{
*Dipartimento di Matematica, Università Roma Tre, Largo S. Murialdo 100146 Roma, Italia. e-mail:

${ }^{\dagger}$ Ecole Normale Supérieure de Lyon, Laboratoire de Physique and CNRS, UMR 5672, 46 Allée d'Italie, 69364 Lyon Cedex 07, France. e-mail: caputo@mat.uniroma3.it, martin@mat.uniroma3.it, fltonine@ens-lyon.fr
} 


\section{Introduction}

Consider simple random walk paths on $\mathbb{Z}$ which start at 0 and end at 0 after $L$ steps, where $L$ is an even integer, i.e. elements of

$$
\Omega_{L}=\left\{\eta \in \mathbb{Z}^{L+1}: \eta_{0}=\eta_{L}=0,\left|\eta_{x+1}-\eta_{x}\right|=1, x=0, \ldots, L-1\right\} .
$$

A well known polymer model (the pinning model) is obtained by assigning to each path $\eta \in \Omega_{L}$ a weight

$$
\lambda^{N(\eta)},
$$

where $\lambda>0$ is a parameter and $N(\eta)$ stands for the number of $x \in\{1, \ldots, L-1\}$ such that $\eta_{x}=0$, i.e. the number of pinned sites. If $\lambda>1$ the weight (1.1) favors pinning of the path whereas if $\lambda<1$ pinning is penalized. The case $\lambda=1$ is referred to as the free case. Normalizing the weights (1.1) one has a probability measure $\mu=\mu_{L}^{\lambda}$ on the set $\Omega_{L}$ of all $\left(\begin{array}{c}L \\ L / 2\end{array}\right)$ paths. This defines our first polymer model.

The second model is obtained by considering only paths that stay non-negative, i.e. elements of

$$
\Omega_{L}^{+}=\left\{\eta \in \Omega_{L}: \eta_{x} \geqslant 0, x=1, \ldots, L-1\right\} .
$$

Normalizing the weights (1.1) one obtains a probability measure $\mu^{+}=\mu_{L}^{+, \lambda}$ on the set $\Omega_{L}^{+}$of all $\frac{2}{L+2}\left(\begin{array}{c}L \\ L / 2\end{array}\right)$ non-negative paths. The positivity constraint will be often referred to as the presence of a wall.
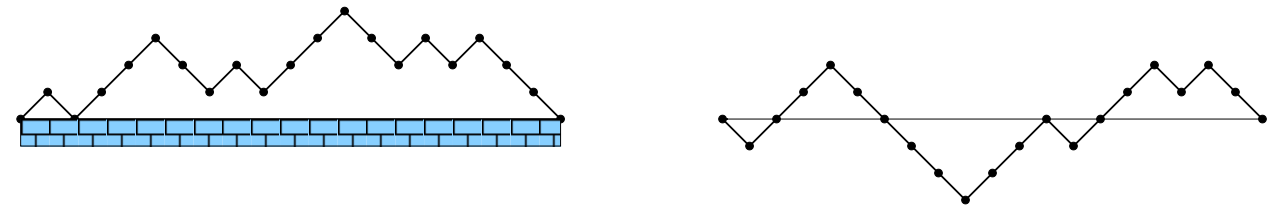

Figure 1: Paths with and without the wall, for $L=20$.

The two models introduced above have been studied for several decades and very precise information is available on their asymptotic properties as $L$ becomes large. The reader is referred to the recent review [14] and references therein and to Section 2 below for more details. For the moment let us briefly recall that both models display a transition from a delocalized to a localized phase as $\lambda$ is increased. Namely, the following scenario holds. For the system without the wall, if $\lambda \leqslant 1$ paths are delocalized (as in the free case $\lambda=1$ ) with $\left|\eta_{L / 2}\right|$ typically of order $\sqrt{L}$ and a vanishing density of pinned sites, while as soon as $\lambda>1$ paths are strongly localized with $\left|\eta_{L / 2}\right|$ typically of order one with a positive density of pinned sites. The critical exponents of the transition can be computed, and the transition itself turns out to be of second order: the fraction of pinned sites goes to zero smoothly when $\lambda \searrow 1$. The system with the wall has a similar behavior but the critical point is $\lambda=2$ instead of $\lambda=1$. Namely, due to the entropic repulsion induced by the wall, a small reward for pinning (as in the case $1<\lambda \leqslant 2$ ) is not sufficient to localize the path.

These models and generalizations thereof, where the simple-random-walk paths are replaced by trajectories of more general Markov chains, are popular tools in the (bio)-physical literature to 
describe, e.g., pinning of polymers on defect lines in different dimensions, the Poland-Scheraga model of DNA denaturation, wetting models,...(we refer for instance to [9], [14, Chap. 1] and references therein).

Presently there is much activity on the quenched disordered version of these models, where the pinning parameter $\lambda$ is replaced by a sequence of (usually log-normal) IID random variables $\lambda_{x}, 0<x<L$. The localization-delocalization transition is present also in this case, and typical questions concern the effect of disorder on the critical point and on the critical exponents (cf. [6], [12], [1] and [21]). Another natural generalization of the polymer models we introduced is to consider $(d+1)$-dimensional interfaces $\left\{\eta_{x}\right\}_{\left\{x \in V \subset \mathbb{Z}^{d}\right\}}$, with or without the hard wall condition $\left\{\eta_{x} \geqslant 0 \forall x \in V\right\}$, and with some pinning interaction (see the recent review [22] and references therein).

We now go back to the two models introduced at the beginning of this section. We are interested in the asymptotic behavior of a continuous time Markov chain naturally associated with them (cf. Figure 2). In the first model - system without the wall - the process is described as follows. Independently, each site $x \in\{1, \ldots, L-1\}$ waits an exponential time with mean one after which the variable $\eta_{x}$ is updated with the following rules:

- if $\eta_{x-1} \neq \eta_{x+1}$, do nothing;

- if $\eta_{x-1}=\eta_{x+1}=j$ and $|j| \neq 1$, set $\eta_{x}=j \pm 1$ with equal probabilities;

- if $\eta_{x-1}=\eta_{x+1}=1$, set $\eta_{x}=0$ with probability $\frac{\lambda}{\lambda+1}$ and $\eta_{x}=2$ otherwise;

- if $\eta_{x-1}=\eta_{x+1}=-1$, set $\eta_{x}=0$ with probability $\frac{\lambda}{\lambda+1}$ and $\eta_{x}=-2$ otherwise.

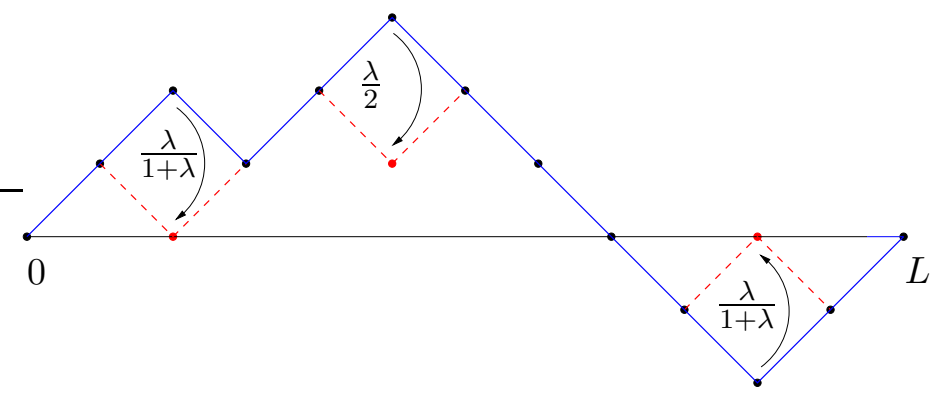

Figure 2: Three possible transitions, with the corresponding rates, for the model without the wall.

This defines an irreducible Markov chain on $\Omega_{L}$ with reversible probability $\mu$. For the system with the wall the process is defined in the same way with the only difference that now if $\eta_{x-1}=$ $\eta_{x+1}=0$ we are forced to keep the value $\eta_{x}=1$. This gives an irreducible Markov chain on $\Omega_{L}^{+}$ with reversible probability $\mu^{+}$.

We shall study the speed at which the equilibria $\mu$ and $\mu^{+}$are approached by our Markov chain mostly by way of estimates on the spectral gap and the mixing time. We refer to Section 2 below 
for the precise definitions, and recall here that the inverse of the spectral gap (also known as relaxation time) measures convergence in the $L^{2}{ }^{-}$norm with respect to the equilibrium measure, while the mixing time measures convergence in total variation norm starting from the worst-case initial condition.

While essentially everything is known about the equilibrium properties of these polymer models, we feel that there is still much to understand as far as the approach to equilibrium is concerned. In particular, one would like to detect the dynamical signature of the phase transition recalled above. Our work is a first attempt in this direction. Before going to a description of our results, we discuss some earlier contributions.

The problem is well understood in the free case $\lambda=1$. In particular, for the system without the wall, the free case is equivalent to the so-called symmetric simple exclusion process which has been analyzed by several authors. We refer to the work of Wilson [23], where among other things the spectral gap of the chain is computed exactly as

$$
\kappa_{L}=1-\cos \left(\frac{\pi}{L}\right)
$$

the principal eigenvalue of the discrete Laplace operator with Dirichlet boundary conditions, and the mixing time $T_{\text {mix }}$ is shown to be of order $L^{2} \log L$ (with upper and lower bounds differing only by a factor 2 in the large $L$ limit).

As far as we know, [18; 17] by Martin and Randall are the only works where the dynamical problem for all $\lambda>0$ was considered. They showed that there is always a polynomial upper bound on the mixing time. Although their proof is carried out in the case of the system with the wall only, their result should apply in the absence of the wall as well. As noted in [17] and as we shall see in detail in the forthcoming sections, for the system with the wall, Wilson's coupling method can be easily modified to prove an upper bound of order $L^{2} \log L$ on the mixing time for all $\lambda \leqslant 1$. On the other hand the problem is harder when $\lambda>1$, and the Markov chain decomposition method of [17] only gives $T_{\text {mix }}=O\left(L^{k}\right)$ for some large non-optimal power $k$.

Let us also mention that, on the non-rigorous or numerical level, various works were devoted recently to the dynamics of polymer models related to the ones we are considering (cf. for instance $[3 ; 2]$ and references therein). These works are mainly motivated by the study of the dynamics of heterogeneous DNA molecules close to the denaturation transition, and therefore focus mainly on the quenched disordered situation. While the dynamics considered there is quite different from the one we study here (and in this sense the results cannot be naturally compared), let us point out that in [3] interesting dynamical transition phenomena are predicted to occur close to the equilibrium phase transition, both for the disordered and for the homogeneous models.

\subsection{Quick survey of our results}

We refer to Section 3 below for the precise statements. We start with the system with the wall. A first result here is that for all $\lambda>0$, the spectral gap is bounded below by the gap (1.2) of the free case, i.e. gap $\geqslant \kappa_{L} \sim \pi^{2} / 2 L^{2}$. Also, we prove that for all $\lambda>0$ the mixing time satisfies $T_{\text {mix }}=O\left(L^{2} \log L\right)$. Furthermore we can prove that these estimates are optimal (up to constant factors) in the delocalized phase, i.e. we can exhibit complementary bounds for $\lambda \leqslant 2$ on the gap and for $\lambda<2$ on the mixing time. In the localized phase $(\lambda>2)$ we expect the relaxation to occur faster than in the free case. However, we prove a general lower 
bound on the mixing time giving $T_{\text {mix }}=\Omega\left(L^{2}\right)$ (we recall that by definition $f(x)=\Omega(g(x)$ ) for $x \rightarrow \infty$ if $\liminf _{x \rightarrow \infty} f(x) / g(x)>0$ ). Concerning the spectral gap we show an upper bound gap $=O\left(L^{-1}\right)$. We conjecture these last two estimates to be of the correct order but a proof of the complementary bounds remains a challenging open problem ${ }^{1}$ (except for $\lambda=\infty$, where we can actually prove that $c_{1} L^{2} \leqslant T_{\text {mix }} \leqslant c_{2} L^{2}$ ).

The fact that the mixing time grows in every situation at least like $L^{2}$ does not exclude that, starting from a particular configuration, the dynamics can relax to equilibrium much faster. In the localized phase we explicitly identify such a configuration and show that the dynamics started from it relaxes within a time $O(\log L)^{3}$.

Concerning the system without the wall we can show that for all $\lambda>1$ the relaxation is at least as fast as in the free case, i.e. gap $\geqslant \kappa_{L}$ and $T_{\text {mix }}=O\left(L^{2} \log L\right)$. However, for $\lambda>1$ we believe the true behavior to be the same as described above for $\lambda>2$ in the presence of the wall. On the other hand, the case $\lambda<1$ is very different from the system with the wall. Here we prove that the spectral gap is no larger than $O\left(L^{-5 / 2}\right)$, up to logarithmic corrections, establishing a clear dynamical transition from localized to delocalized phase. Describing the correct asymptotics of the gap (and of the mixing time) for $\lambda<1$ remains an open problem, although a heuristic argument (see Section 6.1) suggests that the $O\left(L^{-5 / 2}\right)$ behavior may well be the correct one.

Finally, besides focusing on global quantities like gap and mixing time, it is of interest to study how local observables, e.g. the local height function $\eta_{x}$, relax to equilibrium. Note that this point of view is closer to the one of the theoretical physics papers $[3 ; 2]$ we mentioned above. This question is particularly interesting in the localized phase, where the infinite-volume equilibrium measure is the law of a positive recurrent Markov chain and $\eta_{x}$ is of order one. As a consequence of the fact that the spectral gap vanishes for $L \rightarrow \infty$ as an inverse power of $L$, we will show in Theorem 3.6 upper and lower bounds of stretched exponential type for the relaxation of local functions.

\footnotetext{
${ }^{1}$ After this work was completed we were able to prove upper and lower bounds on the spectral gap of order $L^{-1}$ at least in the perturbative regime $\lambda=\Omega\left(L^{4}\right)$. This is part of further work (in progress) on the dynamical aspects of the localization/delocalization transition
} 


\begin{tabular}{|c|c|c|c|}
\hline \multicolumn{4}{|c|}{ Model } \\
\hline parameter & $\begin{array}{c}\text { conjectured } \\
\text { behavior }\end{array}$ & $\begin{array}{c}\text { rigorous } \\
\text { lower bound }\end{array}$ & $\begin{array}{c}\text { rigorous } \\
\text { upper bound }\end{array}$ \\
\hline \multicolumn{4}{|c|}{ Wall, $\lambda<2$} \\
\hline $\begin{array}{l}\text { spectral gap } \\
\text { mixing time }\end{array}$ & $\begin{array}{c}L^{-2} \\
L^{2} \log L\end{array}$ & $\begin{array}{c}L^{-2} \\
L^{2} \log L\end{array}$ & $\begin{array}{c}L^{-2} \\
L^{2} \log L\end{array}$ \\
\hline \multicolumn{4}{|c|}{ Wall, $\lambda=2$} \\
\hline $\begin{array}{l}\text { spectral gap } \\
\text { mixing time }\end{array}$ & $\begin{array}{c}L^{-2} \\
L^{2} \log L\end{array}$ & $\begin{array}{c}L^{-2} \\
L^{2}\end{array}$ & $\begin{array}{c}L^{-2} \\
L^{2} \log L\end{array}$ \\
\hline \multicolumn{4}{|c|}{ Wall, $\lambda>2$} \\
\hline $\begin{array}{l}\text { spectral gap } \\
\text { mixing time }\end{array}$ & $\begin{array}{c}L^{-1} \\
L^{2}\end{array}$ & $\begin{array}{c}L^{-2} \\
L^{2}\end{array}$ & $\begin{array}{c}L^{-1} \\
L^{2} \log L\end{array}$ \\
\hline \multicolumn{4}{|c|}{ No wall, $\lambda<1$} \\
\hline $\begin{array}{l}\text { spectral gap } \\
\text { mixing time }\end{array}$ & $L^{-5 / 2}$ & $L^{5 / 2}(\log L)^{-8}$ & $L^{-5 / 2}(\log L)^{8}$ \\
\hline \multicolumn{4}{|c|}{ No wall, $\lambda=1$} \\
\hline $\begin{array}{l}\text { spectral gap } \\
\text { mixing time }\end{array}$ & $\begin{array}{c}L^{-2} \\
L^{2} \log L\end{array}$ & $\begin{array}{c}L^{-2} \\
L^{2} \log L\end{array}$ & $\begin{array}{c}L^{-2} \\
L^{2} \log L\end{array}$ \\
\hline \multicolumn{4}{|c|}{ No wall, $\lambda>1$} \\
\hline $\begin{array}{l}\text { spectral gap } \\
\text { mixing time }\end{array}$ & $\begin{array}{c}L^{-1} \\
L^{2}\end{array}$ & $\begin{array}{c}L^{-2} \\
L^{2}\end{array}$ & $\begin{array}{c}L^{-1} \\
L^{2} \log L\end{array}$ \\
\hline \multicolumn{4}{|c|}{ Wall/No wall, $\lambda=+\infty$} \\
\hline mixing time & $L^{2}$ & $L^{2}$ & $L^{2}$ \\
\hline
\end{tabular}

Table 1: Rough summary of spectral gap and mixing time bounds. All the entries in the table have to be understood as valid up to multiplicative constants independent of $L$. The statements of our theorems clarify whether the bounds hold with constants depending on $\lambda$ or not. Blank entries in the table correspond to questions which have not been addressed in this work.

The work is organized as follows: in Section 2.1 the model is defined and some basic equilibrium properties are recalled; in Section 2.2 we introduce our dynamics and for completeness we define a few standard tools (spectral gap, mixing time, etc.); in Section 2.3 we describe a basic coupling argument due to D. Wilson [23], which we use at various occasions; in Section 3 we state our main results, which are then proven in Sections 4 to 7 . 


\section{Setup and preliminaries}

In this section we set the notation and collect several tools to be used repeatedly in the rest of the paper.

\subsection{Some equilibrium properties}

Fix $\lambda>0$ and $L \in 2 \mathbb{N}$ and write $\Lambda:=\{0, \ldots, L\}$. As in the introduction $\mu=\mu_{L}^{\lambda}$ denotes the equilibrium measure of the unconstrained system. The Boltzmann weight associated to a configuration $\eta \in \Omega_{L}$ is

$$
\mu_{L}^{\lambda}(\eta):=\frac{\lambda^{N(\eta)}}{Z_{L}(\lambda)}
$$

where $N(\eta):=\#\left\{0<x<L: \eta_{x}=0\right\}$ and

$$
Z_{L}(\lambda):=\sum_{\eta \in \Omega_{L}} \lambda^{N(\eta)}
$$

The equilibrium of the constrained system is described by $\mu^{+}=\mu_{L}^{+, \lambda}$. Here the Boltzmann weight associated to a configuration $\eta \in \Omega_{L}^{+}$is

$$
\mu_{L}^{+, \lambda}(\eta):=\frac{\lambda^{N(\eta)}}{Z_{L}^{+}(\lambda)}
$$

where

$$
Z_{L}^{+}(\lambda):=\sum_{\eta \in \Omega_{L}^{+}} \lambda^{N(\eta)} .
$$

When there is no danger of confusion, we will omit the indexes $\lambda$ and $L$ and write $\mu$ for $\mu_{L}^{\lambda}$ and $\mu^{+}$for $\mu_{L}^{+, \lambda}$.

Considering reflections of the path between consecutive zeros one obtains the following identity:

$$
2 Z_{L}^{+}(2 \lambda)=Z_{L}(\lambda)
$$

Moreover, if $\zeta(\eta):=\left\{x \in \Lambda: \eta_{x}=0\right\}$ is the set of zeros of the configuration $\eta$, one has

$$
\mu_{L}^{+, 2 \lambda}(\zeta=S)=\mu_{L}^{\lambda}(\zeta=S), \quad S \subset \Lambda .
$$

In other words, the thermodynamic properties of the two models are essentially equivalent modulo a change of $\lambda$. On the other hand, we will see that the two present very different dynamical phenomena. 


\subsubsection{Free energy and the localization/delocalization transition}

Let $\mathbf{P}$ and $\mathbf{E}$ denote the law and expectation of the one-dimensional simple random walk $\eta:=$ $\left\{\eta_{n}\right\}_{n} \geqslant 0$ with initial condition $\eta_{0}=0$. Then,

$$
Z_{L}(\lambda)=2^{L} \mathbf{E}\left(\lambda^{N(\eta)} 1_{\left\{\eta_{L}=0\right\}}\right),
$$

and

$$
Z_{L}^{+}(\lambda)=2^{L} \mathbf{E}\left(\lambda^{N(\eta)} 1_{\left\{\eta_{L}=0\right\}} 1_{\left\{\eta_{x} \geqslant 0 \forall x<L\right\}}\right) .
$$

The free energy is defined for the system without the wall as

$$
F(\lambda):=\lim _{L \rightarrow \infty} \frac{1}{L} \log Z_{L}(\lambda)-\log 2 .
$$

The limit exists by super-additivity. Similarly, the free energy of the system with the wall is denoted by $F^{+}(\lambda)$. Of course, one has $F^{+}(\lambda)=F(\lambda / 2)$, as follows from (2.5).

The following is well known (cf. e.g. [14, Ch. 2]): $F(\lambda)=0$ for $\lambda \leqslant 1$ and $F(\lambda)>0$ for $\lambda>1$. Moreover, for $\lambda>1, F(\lambda)$ can be equivalently defined as the unique positive solution of

$$
\sum_{n \in 2 \mathbb{N}} \mathbf{P}\left(\inf \left\{k>0: \eta_{k}=0\right\}=n\right) e^{-n F}=\frac{1}{\lambda} .
$$

Together with the explicit expression for the Laplace transform of the first return time of the simple random walk,

$$
\sum_{n \in 2 \mathbb{N}} z^{n} \mathbf{P}\left(\inf \left\{k>0: \eta_{k}=0\right\}=n\right)=1-\sqrt{1-z^{2}}
$$

for $|z| \leqslant 1,(2.10)$ implies

$$
F(\lambda)=\frac{1}{2} \log \left[\frac{\lambda^{2}}{2 \lambda-1}\right]
$$

for $\lambda>1$. Note that $F^{+}(\lambda)>0$ if and only if $\lambda>2$.

We will need the following sharp estimates on the asymptotic behavior of the partition function for large $L$ :

Theorem 2.1. [14, Th. 2.2]

$$
2^{-L} Z_{L}(\lambda) \stackrel{L \rightarrow \infty}{\sim} C(\lambda) \times\left\{\begin{array}{lll}
e^{L F(\lambda)} & \text { for } \lambda>1 \\
L^{-1 / 2} & \text { for } \lambda=1 \\
L^{-3 / 2} & \text { for } \lambda<1
\end{array}\right.
$$

where $C(\lambda)>0$ for every $\lambda$, i.e. the ratio of the two sides in (2.13) converges to one. 
We refer to [14, Th. 2.2] for an expression of $C(\lambda)$ in terms of the law $\mathbf{P}(\cdot)$. From the explicit expression (2.12) one sees that $F(\cdot)$ is differentiable with respect to $\lambda$ in $(0, \infty)$. Since the free energy is a convex function of $\log \lambda$, one deduces that the average density of pinned sites satisfies

$$
\lim _{L \rightarrow \infty} \frac{1}{L} \mu_{L}^{\lambda}(N(\eta))=\frac{d F(\lambda)}{d \log \lambda}\left\{\begin{array}{lll}
=0 & \text { if } \quad \lambda \leqslant 1 \\
>0 & \text { if } \quad \lambda>1
\end{array}\right.
$$

For this reason, one calls the region of parameters $\lambda \leqslant 1$ delocalized phase and $\lambda>1$ localized phase, and $\lambda=1$ the critical point (for the system with the wall, the critical point is therefore $\lambda=2)$.

One can go much beyond the density statement (2.14) in characterizing the two phases. In the rest of this section we recall some known results.

\subsubsection{The strictly delocalized phase}

This terminology refers to the situation $\lambda<1$ (or $\lambda<2$ with the wall). In this, case, the number of zeros $N(\eta)$ is typically finite and its law has an exponential tail. In what follows we write $c=c(\lambda)$ for a positive constant (not necessarily the same at each occurrence) which can depend on $\lambda$ but not on $L$. There exists $c=c(\lambda)$ such that

$$
\mu_{L}^{\lambda}(N(\eta) \geqslant j) \leqslant c e^{-j / c},
$$

uniformly in $L$. (This simply follows from

$$
\mu_{L}^{\lambda}(N(\eta) \geqslant j) \leqslant e^{-\varepsilon j} \mu_{L}^{\lambda}\left(e^{\varepsilon N(\eta)}\right)=e^{-\varepsilon j} \frac{Z_{L}\left(\lambda e^{\varepsilon}\right)}{Z_{L}(\lambda)},
$$

if we choose $\varepsilon>0$ small enough so that $\lambda \exp (\varepsilon)<1$, cf. Theorem 2.1.) It is also easy to see that there is a non-zero probability that $N(\eta)=0$ :

$$
\mu_{L}^{\lambda}(N(\eta)=0)=2 \frac{\mathbf{P}\left(\eta_{L}=0, \eta_{x}>0 \forall 1<x<L\right)}{2^{-L} Z_{L}^{\lambda}} \stackrel{L \rightarrow \infty}{\sim} c \in(0,1)
$$

where in the last step we used (2.13) and the fact that

$$
\lim _{L \rightarrow \infty} L^{3 / 2} \mathbf{P}\left(\eta_{L}=0, \eta_{x}>0 \forall 1<x<L\right)>0,
$$

[8, Sec. III.3]. Finally, we will need the following upper bound on the probability that there exists a zero far away from the boundaries of the system:

$$
\mu_{L}^{\lambda}\left(\exists x: \ell \leqslant x \leqslant L-\ell, \eta_{x}=0\right) \leqslant \frac{c}{\ell^{1 / 2}},
$$

for every $L$ and $\ell<L / 2$. This can be extracted immediately from Theorem 2.1. 


\subsubsection{The localized phase}

Here $\lambda>1$ for the system without the wall or $\lambda>2$ with the wall. In the localized phase, $\left|\eta_{x}\right|$ is typically of order 1 with exponential tails, and correlation functions between local functions decay exponentially fast. Given a function $f: \Omega_{L} \rightarrow \mathbb{R}$ we denote by $\mathcal{S}_{f}$ the support of $f$, i.e. the minimal set $I \in \Lambda$ such that $f$ depends only on $\left\{\eta_{x}\right\}_{x \in I}$, and set $\|f\|_{\infty}:=\max _{\eta \in \Omega_{L}}|f(\eta)|$. Then, it is not difficult to prove:

Lemma 2.2. Let $\lambda>1$. For every $L \in 2 \mathbb{N}$ and $x, \ell \leqslant L$

$$
\mu_{L}^{\lambda}\left(\left|\eta_{x}\right| \geqslant \ell\right) \leqslant c e^{-\ell F(\lambda)} .
$$

Moreover, for every pair of functions $f, g: \Omega_{L} \rightarrow \mathbb{R}$

$$
\left|\mu_{L}^{\lambda}(f g)-\mu_{L}^{\lambda}(f) \mu_{L}^{\lambda}(g)\right| \leqslant c\|f\|_{\infty}\|g\|_{\infty} e^{-d\left(\mathcal{S}_{f}, \mathcal{S}_{g}\right) / c}
$$

where $d(\cdot, \cdot)$ denotes the usual distance between subsets of $\mathbb{Z}$. One has exponential loss of memory of boundary conditions:

$$
\sup _{L>k}\left|\mu_{L}^{\lambda}(f)-\mu_{k}^{\lambda}(f)\right| \leqslant c\|f\|_{\infty} e^{-d\left(\mathcal{S}_{f},\{k\}\right) / c},
$$

where $d\left(\mathcal{S}_{f},\{k\}\right)$ is the distance between $\mathcal{S}_{f} \subset\{0, \ldots, k\}$ and the point $\{k\}$. Finally, for every bounded local function the thermodynamic limit

$$
\lim _{L \rightarrow \infty} \mu_{L}^{\lambda}(f)
$$

exists. The same holds for $\mu_{L}^{+, \lambda}$ if $\lambda>2$.

These results follow for instance from those proven in [13] in a more general context, i.e. when the constant $\lambda$ is replaced by a sequence of IID random variables $\lambda_{x}, x \in \Lambda$.

\subsection{The Markov chain}

The process described in the introduction is nothing but the standard heat bath dynamics. For the system without the wall we can formulate this as follows. Let $Q_{x}$ denote the $\mu$-conditional

expectation at $x$ given the values of the heights $\eta_{y}$ at all vertices $y \neq x$, where $\mu=\mu_{L}^{\lambda}$ is the equilibrium measure (2.1). Namely, for all $f: \Omega_{L} \rightarrow \mathbb{R}$, and $x \in\{1, \ldots, L-1\}$ we write

$$
Q_{x} f=\mu\left(f \mid \eta_{y}, y \neq x\right) .
$$

Our process is then the continuous-time Markov chain with infinitesimal generator given by

$$
\mathcal{L} f=\sum_{x=1}^{L-1}\left[Q_{x} f-f\right], \quad f: \Omega_{L} \rightarrow \mathbb{R} .
$$

Note that the generator can be written in more explicit terms as

$$
\mathcal{L} f(\eta)=\sum_{x=1}^{L-1} c_{x}(\eta)\left[f\left(\eta^{x}\right)-f(\eta)\right],
$$


where $\eta^{x}$ denotes the configuration $\eta$ after the $x$-th coordinate has been "flipped", and the rates $c_{x}(\eta)$ are given by

$$
c_{x}(\eta)= \begin{cases}\frac{1}{2} & \eta_{x-1}=\eta_{x+1} \notin\{-1,1\} \\ \frac{\lambda}{\lambda+1} & \left(\eta_{x-1}, \eta_{x}, \eta_{x+1}\right)=(1,2,1) \text { or }(-1,-2,-1) \\ \frac{1}{\lambda+1} & \left(\eta_{x-1}, \eta_{x}, \eta_{x+1}\right)=(1,0,1) \text { or }(-1,0,-1) \\ 0 & \eta_{x-1} \neq \eta_{x+1}\end{cases}
$$

We shall write $P_{t}, t \geqslant 0$, for the associated semigroup acting on functions on $\Omega_{L}$. Given an initial condition $\xi$, we write $\eta^{\xi}(t)$ for the configuration at time $t$, so that the expected value of $f\left(\eta^{\xi}(t)\right)$ can be written as $P_{t} f(\xi)$.

Similarly, in the presence of the wall, if $Q_{x}^{+}$denotes the $\mu^{+}$-conditional expectation at $x$ given the path at all vertices $y, y \neq x$, where $\mu^{+}=\mu_{L}^{+, \lambda}$ is the equilibrium measure (2.3), the infinitesimal generator becomes

$$
\mathcal{L}^{+} f=\sum_{x=1}^{L-1}\left[Q_{x}^{+} f-f\right], \quad f: \Omega_{L}^{+} \rightarrow \mathbb{R} .
$$

We write $\eta^{+, \xi}(t)$ for the configuration at time $t$ with initial condition $\xi$. Similarly, we write $P_{t}^{+}$ for the associated semigroups acting on functions on $\Omega_{L}^{+}$. If no confusion arises we shall drop the + superscript and use again the notation $\eta^{\xi}(t), P_{t}$ as in the case without the wall.

\subsubsection{Coupling and monotonicity}

A standard procedure allows to define a probability measure $\mathbb{P}$ which is a simultaneous coupling of the laws of processes associated to different initial conditions. Moreover, the measure $\mathbb{P}$ can be used to couple the laws of processes corresponding to different values of $\lambda$ and to couple paths evolving with the wall to paths evolving without the wall.

The construction of $\mathbb{P}$, the global coupling, can be described as follows. We need $L-1$ independent Poisson processes $\omega_{x}$ with parameter 1 , which mark the updating times at each $x \in\{1, \ldots, L-1\}$, and a sequence $\left\{u_{n}, n \in \mathbb{N}\right\}$ of independent random variables with uniform distribution in $[0,1]$, which stand for the "coins" to be flipped for the updating choices. Given an initial condition $\xi$, a realization $\omega$ of the Poisson processes and a realization $u$ of the variables $u_{n}$ we can compute the path $\eta^{\xi}(s), s \leqslant t$, for any fixed $t>0$, as follows: sites to be updated together with their updating times up to time $t$ are chosen according to $\omega$; if the $k$-th update occurs at site $x$ and at time $s_{k}$, and $\eta_{x-1}^{\xi}\left(s_{k}\right)=\eta_{x+1}^{\xi}\left(s_{k}\right)=j$ then

- if $|j| \neq 1$, set $\eta_{x}=j+1$ if $u_{k} \leqslant \frac{1}{2}$, and $\eta_{x}=j-1$ otherwise;

- if $j=1$, set $\eta_{x}=0$ if $u_{k} \leqslant \frac{\lambda}{\lambda+1}$, and $\eta_{x}=2$ otherwise;

- if $j=-1$, set $\eta_{x}=0$ if $u_{k} \leqslant \frac{\lambda}{\lambda+1}$, and $\eta_{x}=-2$ otherwise.

Of course, in case of an evolution with the wall we have to add the constraint that a site $x$ such that $\eta_{x-1}^{\xi}\left(s_{k}\right)=\eta_{x+1}^{\xi}\left(s_{k}\right)=0$ cannot change.

We can run this process for any initial data $\xi$. It is standard to check that, provided we use the same realization $(\omega, u)$ for all copies, the above construction produces the desired coupling. 
Given two paths $\xi, \sigma \in \Omega_{L}$ we say that $\xi \leqslant \sigma$ iff $\xi_{x} \leqslant \sigma_{x}$ for all $x \in \Lambda$. By construction, if $\xi \leqslant \sigma$, then $\mathbb{P}$-a.s. we must have $\eta^{\xi}(t) \leqslant \eta^{\sigma}(t)$ at all times. The same holds for the evolution with the wall. In particular, we will be interested in the evolution started from the maximal path $\wedge$, defined as $\wedge_{x}=x$ for $x \leqslant L / 2$ and $\wedge_{x}=L-x$ for $L / 2 \leqslant x \leqslant L$, and from the minimal path $\vee:=-\wedge$. For the system with the wall the minimal path is the zigzag line given by $\eta_{x}=0$ for all even $x$ and $\eta_{x}=1$ for all odd $x$. For simplicity, we shall again use the notation $\vee$ for this path.

Note that if the initial condition $\xi$ evolves with the wall while $\sigma$ evolves without the wall we have $\eta^{\sigma}(t) \leqslant \eta^{+, \xi}(t)$, if $\sigma \leqslant \xi$. Finally, for evolutions with the wall we have an additional monotonicity in $\lambda$, i.e. if $\sigma$ evolves with parameter $\lambda$ and $\xi$ with parameter $\lambda^{\prime}$ then $\eta^{+, \sigma}(t) \leqslant \eta^{+, \xi}(t)$ if $\sigma \leqslant \xi$ and $\lambda \geqslant \lambda^{\prime}$.

Let $\mathbb{E}$ denote expectation with respect to the global coupling $\mathbb{P}$. Using the notation $\mathbb{E}\left[f\left(\eta^{\xi}(t)\right)\right]=$ $P_{t} f(\xi)$ the monotonicity discussed in the previous paragraph takes the form of the statement that for every fixed $t \geqslant 0$, the function $P_{t} f$ is increasing whenever $f$ is increasing, where a function $f$ is called increasing if $f(\xi) \geqslant f(\sigma)$ for any $\sigma, \xi$ such that $\sigma \leqslant \xi$. A whole family of so-called FKG inequalities can be derived from the global coupling. For instance, the comparison between different $\lambda$ 's mentioned above, by taking the limit $t \rightarrow \infty$ yields the inequality $\mu^{+, \lambda}(f) \leqslant \mu^{+, \lambda^{\prime}}(f)$, valid for any increasing $f$ and any $\lambda \geqslant \lambda^{\prime}$. Also, a straightforward modification of the same argument proves that for any subset $S \subset \Lambda$ and any pair of paths $\sigma, \xi \in \Omega_{L}$ such that $\sigma \leqslant \xi$, then

$$
\mu(f \mid \eta=\sigma \text { on } S) \leqslant \mu(f \mid \eta=\xi \text { on } S),
$$

for every increasing $f: \Omega_{L} \rightarrow \mathbb{R}$. The same arguments apply in the presence of the wall, giving (2.27) with $\mu^{+}$in place of $\mu$, for every increasing $f: \Omega_{L}^{+} \rightarrow \mathbb{R}$.

We would like to stress that monotonicity and its consequences such as FKG inequalities play an essential role in the analysis of our models. Unfortunately, these nice properties need not be available in other natural polymer models.

\subsubsection{Spectral gap and mixing time}

To avoid repetitions we shall state the following definitions for the system without the wall only (otherwise simply replace $\mu$ by $\mu^{+}, \mathcal{L}$ by $\mathcal{L}^{+}$etc. in the expressions below).

Let $P_{t}\left(\xi, \xi^{\prime}\right)=\mathbb{P}\left(\eta^{\xi}(t)=\xi^{\prime}\right)$ denote the kernel of our Markov chain. It is easily checked that $P_{t}$ satisfies reversibility with respect to $\mu$, i.e.

$$
\mu(\xi) P_{t}\left(\xi, \xi^{\prime}\right)=\mu\left(\xi^{\prime}\right) P_{t}\left(\xi^{\prime}, \xi\right), \quad \xi, \xi^{\prime} \in \Omega_{L},
$$

or, in other terms, $\mathcal{L}$ and $P_{t}$ are self-adjoint in $L^{2}(\mu)$. In particular, $\mu$ is the unique invariant distribution and $P_{t}(\xi, \eta) \rightarrow \mu(\eta)$ as $t \rightarrow \infty$ for every $\xi, \eta \in \Omega_{L}$. The rate at which this convergence takes place will be measured using the following standard tools.

The Dirichlet form associated to (2.25) is:

$$
\mathcal{E}(f, f)=-\mu(f \mathcal{L} f)=\sum_{0<x<L} \mu\left[\left(Q_{x} f-f\right)^{2}\right] .
$$


The spectral gap is defined by

$$
\operatorname{gap}=\inf _{f: \Omega_{L} \rightarrow \mathbb{R}} \frac{\mathcal{E}(f, f)}{\operatorname{Var}(f)}
$$

where $\operatorname{Var}(f)=\mu\left(f^{2}\right)-\mu(f)^{2}$ denotes the variance. The spectral gap is the smallest non-zero eigenvalue of $-\mathcal{L}$. It measures the rate of exponential decay of the variance of $P_{t} f$ as $t \rightarrow \infty$, i.e. gap is the (optimal) constant such that for any $f, t>0$ :

$$
\operatorname{Var}\left(P_{t} f\right) \leqslant e^{-2 t \text { gap }} \operatorname{Var}(f) .
$$

The mixing time $T_{\text {mix }}$ is defined by

$$
T_{\text {mix }}=\inf \left\{t>0: \max _{\xi \in \Omega_{L}}\left\|P_{t}(\xi, \cdot)-\mu\right\|_{\text {var }} \leqslant 1 / e\right\},
$$

where $\|\cdot\|_{\text {var }}$ stands for the usual total variation norm:

$$
\left\|\nu-\nu^{\prime}\right\|_{\mathrm{var}}=\frac{1}{2} \sum_{\eta \in \Omega_{L}}\left|\nu(\eta)-\nu^{\prime}(\eta)\right|,
$$

for arbitrary probabilities $\nu, \nu^{\prime}$ on $\Omega_{L}$. We refer e.g. to Peres [20] for more background on mixing times. Using familiar relations between total variation distance and coupling and using the monotonicity of our Markov chain we can estimate, for any $\xi$ and $t>0$ :

$$
\left\|P_{t}(\xi, \cdot)-\mu\right\|_{\text {var }} \leqslant \mathbb{P}\left(\eta^{\wedge}(t) \neq \eta^{\vee}(t)\right),
$$

where $\eta^{\wedge}(t), \eta^{\vee}(t)$ denote the evolutions from maximal and minimal paths respectively. This will be our main tool in estimating $T_{\text {mix }}$ from above. Also, (2.33) will be used to estimate the spectral gap from below. Indeed, a standard argument (see e.g. Proposition 3 in [23]) shows that $-\liminf _{t \rightarrow \infty} \frac{1}{t} \log \left(\max _{\xi}\left\|P_{t}(\xi, \cdot)-\mu\right\|_{\text {var }}\right)$ is a lower bound on the gap, so that

$$
\operatorname{gap} \geqslant-\liminf _{t \rightarrow \infty} \frac{1}{t} \log \mathbb{P}\left(\eta^{\wedge}(t) \neq \eta^{\vee}(t)\right) .
$$

Finally, it is well known that gap and $T_{\text {mix }}$ satisfy the general relations

$$
\operatorname{gap}^{-1} \leqslant T_{\text {mix }} \leqslant \operatorname{gap}^{-1}\left(1-\log \mu_{*}\right),
$$

where $\mu_{*}=\min _{\eta} \mu(\eta)$. Note that in our case $-\log \mu_{*}=O(L)$ for every fixed $\lambda$.

\subsection{A first argument}

Let $\Delta$ denote the discrete Laplace operator

$$
(\Delta \varphi)_{x}=\frac{1}{2}\left(\varphi_{x-1}+\varphi_{x+1}\right)-\varphi_{x} .
$$

We shall need the following computation in the sequel. 
Lemma 2.3. Set $\delta=2 /(1+\lambda)$. For the system without the wall, for every $x=1, \ldots, L-1$ :

$$
\mathcal{L} \eta_{x}=(\Delta \eta)_{x}+(1-\delta) 1_{\left\{\eta_{x-1}=\eta_{x+1}=-1\right\}}-(1-\delta) 1_{\left\{\eta_{x-1}=\eta_{x+1}=1\right\}} .
$$

For the system with the wall, for every $x=1, \ldots, L-1$ :

$$
\mathcal{L}^{+} \eta_{x}=(\Delta \eta)_{x}+1_{\left\{\eta_{x-1}=\eta_{x+1}=0\right\}}-(1-\delta) 1_{\left\{\eta_{x-1}=\eta_{x+1}=1\right\}}
$$

If $\lambda=1$, then $\delta=1$ so that (2.36) has pure diffusive character. If $\lambda \neq 1$ the correction terms represent the attraction $(\lambda>1)$ or repulsion $(\lambda<1)$ at 0 . In the presence of the wall there is an extra repulsive term.

Proof. From (2.25) we see that $\mathcal{L} \eta_{x}=\mu\left[\eta_{x} \mid \eta_{x-1}, \eta_{x+1}\right]-\eta_{x}$. If $\eta_{x-1} \neq \eta_{x+1}$ then $\mu\left[\eta_{x} \mid \eta_{x-1}, \eta_{x+1}\right]=\frac{1}{2}\left(\eta_{x-1}+\eta_{x+1}\right)$. The same holds if $\eta_{x-1}=\eta_{x+1}=j$ with $|j| \neq 1$. Finally, if $\eta_{x-1}=\eta_{x+1}= \pm 1$ we have that

$$
\mu\left[\eta_{x} \mid \eta_{x-1}, \eta_{x+1}\right]= \pm \delta=\delta \frac{1}{2}\left(\eta_{x-1}+\eta_{x+1}\right) .
$$

This proves (2.36). The proof of $(2.37)$ is the same, with the observation that

$$
\mu\left[\eta_{x} \mid \eta_{x-1}, \eta_{x+1}\right]=1
$$

if $\eta_{x-1}=\eta_{x+1}=0$.

Next, we describe an argument which is at the heart of Wilson's successful analysis of the free case $\lambda=1$. Define the non-negative profile function $g_{x}:=\sin \left(\frac{\pi x}{L}\right)$ and observe that $g$ satisfies

$$
(\Delta g)_{x}=-\kappa_{L} g_{x}, \quad x \in\{1, \ldots, L-1\}
$$

where $\kappa_{L}$ is the first Dirichlet eigenvalue of $\Delta$ given in (1.2). Define

$$
\Phi(\eta)=\sum_{x=1}^{L-1} g_{x} \eta_{x}
$$

Lemma 2.3 shows that for $\lambda=1$, for the system without the wall, one has

$$
\mathcal{L} \Phi=\sum_{x=1}^{L-1} g_{x}(\Delta \eta)_{x}=\sum_{x=1}^{L-1}(\Delta g)_{x} \eta_{x}=-\kappa_{L} \Phi,
$$

where we use summation by parts and (2.38). Therefore $P_{t} \Phi(\eta)=e^{-\kappa_{L} t} \Phi(\eta)$ for all $t$ and $\eta$. In particular, if we define

$$
\widetilde{\Phi}_{t}=\sum_{x=1}^{L-1} g_{x}\left(\eta_{x}^{\wedge}(t)-\eta_{x}^{\vee}(t)\right),
$$

then $\mathbb{E} \widetilde{\Phi}_{t}=P_{t} \Phi(\wedge)-P_{t} \Phi(\vee)=\widetilde{\Phi}_{0} e^{-\kappa_{L} t}$. Note that monotonicity implies that $\widetilde{\Phi}_{t} \geqslant 0$ for all $t \geqslant 0$. Since $g_{x} \geqslant \sin (\pi / L), 0<x<L$, we have

$$
\begin{aligned}
\mathbb{P}\left(\eta^{\wedge}(t) \neq \eta^{\vee}(t)\right) & \leqslant \mathbb{P}\left(\widetilde{\Phi}_{t} \geqslant 2 \sin (\pi / L)\right) \\
& \leqslant \frac{\mathbb{E} \widetilde{\Phi}_{t}}{2 \sin (\pi / L)}=\frac{\widetilde{\Phi}_{0} e^{-\kappa_{L} t}}{2 \sin (\pi / L)}
\end{aligned}
$$


Inserting (2.42) in (2.34) one obtains

$$
\text { gap } \geqslant \kappa_{L}
$$

(Since here $\mathcal{L} \Phi=-\kappa_{L} \Phi$ this actually gives gap $=\kappa_{L}$.) Using (2.33) one has the upper bound $T_{\text {mix }} \leqslant \kappa_{L}^{-1} \log \frac{e \widetilde{\Phi}_{0}}{2 \sin (\pi / L)}$. Since $\kappa_{L} \sim \pi^{2} / 2 L^{2}$ and $\widetilde{\Phi}_{0} \leqslant L^{2} / 2$, we have

$$
T_{\text {mix }} \leqslant\left(\frac{6}{\pi^{2}}+o(1)\right) L^{2} \log L
$$

The estimate (2.44) is of the correct order in $L$, although the constant might be off by a factor 6 , cf. Wilson's work [23] for more details.

\section{Main results}

\subsection{Spectral gap and mixing time with the wall}

The first result shows that relaxation will never be slower than in the free case without the wall.

Theorem 3.1. For every $\lambda>0$,

$$
\operatorname{gap} \geqslant \kappa_{L}
$$

where $\kappa_{L}=1-\cos \left(\frac{\pi}{L}\right)$. Moreover,

$$
T_{\text {mix }} \leqslant\left(\frac{6}{\pi^{2}}+o(1)\right) L^{2} \log L .
$$

The proof of these estimates will be based on a comparison with the free case, which boils down to a suitable control on the correction terms described in Lemma 2.3. This will be worked out in Section 4.

The next theorem gives complementary bounds which imply that Theorem 3.1 is sharp up to constants in the strictly delocalized phase.

Theorem 3.2. For every $\lambda \leqslant 2$,

$$
\text { gap } \leqslant c L^{-2},
$$

where $c>0$ is independent of $\lambda$ and $L$. Moreover, for $\lambda<2$ we have

$$
T_{\text {mix }} \geqslant\left(\frac{1}{2 \pi^{2}}+o(1)\right) L^{2} \log L .
$$

For $\lambda>2$ we have

$$
\text { gap } \leqslant c L^{-1},
$$

where $c=c(\lambda)$ is independent of $L$. Finally, for every $\lambda>0$ :

$$
T_{\text {mix }} \geqslant c L^{2},
$$

for some $c>0$ independent of $\lambda$ and $L$. 
The proof of the upper bounds (3.3) and (3.5) will be obtained by choosing a suitable test function in the variational principle defining the spectral gap. The estimate (3.4) will be achieved by a suitable comparison with the free case, while (3.6) will follow by a comparison with the extreme case $\lambda=\infty$. These results are proven in Section 5 .

We expect the $L^{2} \log L$ estimate (3.4) to hold at the critical point $\lambda=2$ as well, but for our proof we require strict delocalization (in (3.4) what may depend on $\lambda$ is the $o$ (1) function).

We conjecture the estimates (3.5) and (3.6) to be sharp (up to constants) in the localized phase $\lambda>2$. In particular, in Proposition 5.6 we prove that (3.6) is sharp at $\lambda=\infty$.

It is interesting that, although the mixing time is $\Omega\left(L^{2}\right)$ in every situation, for the model with the wall we can prove that the dynamics converges to the invariant measure much faster if started from the minimal configuration, $\vee$, which so to speak is already "sufficiently close to equilibrium":

Theorem 3.3. For $\lambda>2$ there exists $c(\lambda)<\infty$ such that

$$
\limsup _{\substack{L \rightarrow \infty, t \geqslant c(\lambda)(\log L)^{3}}}\left\|P_{t}(\vee, \cdot)-\mu_{L}^{+, \lambda}\right\|_{\text {var }}=0 .
$$

On the other hand

$$
\liminf _{\substack{L \rightarrow \infty, t \leqslant(\log L)^{2} / c(\lambda)}}\left\|P_{t}(\vee, \cdot)-\mu_{L}^{+, \lambda}\right\|_{\mathrm{var}}=1 .
$$

The proof of Theorem 3.3 can be found in Section 7 .

\subsection{Spectral gap and mixing time without the wall}

We start with the lower bounds on the gap and upper bounds on $T_{\text {mix }}$.

Theorem 3.4. For any $\lambda \geqslant 1$, gap and $T_{\operatorname{mix}}$ satisfy (3.1) and (3.2) respectively.

The proof is somewhat similar to the proof of Theorem 3.1 and it will be given in Section 4 . We turn to the upper bounds on the gap and lower bounds on $T_{\text {mix }}$.

Theorem 3.5. For $\lambda>1$, gap and $T_{\operatorname{mix}}$ satisfy (3.5) and (3.6) respectively. If $\lambda<1$, on the other hand, there exists $c(\lambda)<\infty$ such that

$$
\operatorname{gap} \leqslant c(\lambda) \frac{(\log L)^{8}}{L^{5 / 2}} .
$$

The proof of the first two estimates is essentially as for (3.5) and (3.6), and it is given in Section 5. As in the system with the wall, we believe these estimates to be of the right order in $L$.

The estimate (3.9) shows that relaxation in the strictly delocalized phase is radically different from that of the model with wall. The proof is based on a somewhat subtle analysis of the behavior of the signed area under the path. This will be worked out in Section 6. While the logarithmic correction is spurious it might be that (3.9) captures the correct power law decay of the spectral gap for $\lambda<1$, as argued in Section 6.1 below. Of course, by (2.35) the bound (3.9) implies that $T_{\text {mix }} \geqslant L^{5 / 2} /\left(c(\lambda)(\log L)^{8}\right)$. 


\subsection{Relaxation of local observables in the localized phase}

Finally, we show that in the localized phase local observables decay to equilibrium following a stretched exponential behavior. For technical reasons we restrict to the model with the wall. As it will be apparent from the discussion below, our arguments are similar to the heuristic ones introduced by D. Fisher and D. Huse [10] in the context of low temperature stochastic Ising models (see also the more mathematical papers [4] and [11]). Specifically, bounds on the probability of creating an initial local large fluctuation of the interface around the support of the local function and on the time necessary in order to make it disappear will play a key role.

In the localized phase the infinite-volume measure (denoted by $\mu_{\infty}^{+}$) is the law of a positive recurrent Markov chain. In order to have more natural statements in Theorem 3.6 below, we take the thermodynamic limit as follows. We start from the system with zero boundary conditions at $\pm L$ for $L \in 2 \mathbb{N}$ (instead of $0, L$ as we did until now) and we denote (with a slight abuse of notation) by $\mu_{2 L}^{+, \lambda}$ the corresponding equilibrium measure. Then, for every bounded function $f$ with finite support $\mathcal{S}_{f} \subset \mathbb{Z}$, the limit

$$
\mu_{\infty}^{+}(f):=\lim _{L \rightarrow \infty} \mu_{2 L}^{+, \lambda}(f)
$$

exists (cf. Lemma 2.2 and in particular $(2.22)$ ). Similarly, for any fixed $t \geqslant 0$, if $P_{t, 2 L}^{+}$denotes the semigroup in the system with zero boundary conditions at $\pm L$, we denote by

$$
P_{t} f(\eta):=\lim _{L \rightarrow \infty} P_{t, 2 L}^{+} f(\eta),
$$

the semigroup associated to the infinite-volume dynamics in the localized phase. Standard approximation estimates show that the above pointwise limit is well defined for every bounded local function $f$ (see e.g. the argument in proof of Claim 7.2 below for more details).

Theorem 3.6. For every $\lambda>2$ there exists $m>0$ such that the following holds.

1) For every bounded local function $f$ there exists a constant $C_{f}<\infty$ depending on $\mathcal{S}_{f}$ and $\|f\|_{\infty}$ such that

$$
\operatorname{Var}_{\mu_{\infty}^{+}}\left(P_{t} f\right) \leqslant C_{f} e^{-m t^{1 / 3}}
$$

for every $t \geqslant 0$.

2) For functions $f$ of the form

$$
f^{a}, I(\eta):=1_{\left\{\eta_{x} \leqslant a_{x} \forall x \in I\right\}},
$$

where $I$ is a finite subset of $\mathbb{Z}$ and $a_{x} \in \mathbb{N}$, there exists a constant $c_{f}>0$ such that

$$
\operatorname{Var}_{\mu_{\infty}^{+}}\left(P_{t} f\right) \geqslant c_{f} e^{-\sqrt{t} / m}
$$

for every $t \geqslant 0$.

The fact that the exponents of $t$ in (3.10) and (3.12) do not match is essentially a consequence of the fact that the exponents of $L$ in our upper and lower bounds on the spectral gap in the localized phase also do not match (cf. (3.1) and (3.5)). Theorem 3.6 is proven in Section 7 . 


\section{Proof of Theorem 3.1 and Theorem 3.4}

We are going to use the argument described in Section 2.3. In particular, we recall that both Theorem 3.1 and Theorem 3.4 will follow once we show that

$$
\mathbb{E} \widetilde{\Phi}_{t} \leqslant e^{-\kappa_{L} t} \widetilde{\Phi}_{0}, \quad t>0
$$

where $\widetilde{\Phi}_{t}$ is given by (2.41). Indeed, assuming (4.1) we can repeat the estimates leading to (2.43) and (2.44) without modifications, which achieves the proof.

\subsection{Proof of (4.1) with the wall}

We shall prove that (4.1) holds for the system with the wall, for any $\lambda>0$. Observe that

$$
\frac{d}{d t} \mathbb{E} \widetilde{\Phi}_{t}=\frac{d}{d t} P_{t} \Phi(\wedge)-\frac{d}{d t} P_{t} \Phi(\vee)=P_{t} \mathcal{L} \Phi(\wedge)-P_{t} \mathcal{L} \Phi(\vee)
$$

where, for simplicity, we omit the + superscript and write $\mathcal{L}$ for $\mathcal{L}^{+}$and $P_{t}$ for $P_{t}^{+}$. From Lemma 2.3 and (2.40) we know that

$$
\mathcal{L} \Phi=\sum_{x=1}^{L-1} g_{x} \mathcal{L} \eta_{x}=-\kappa_{L} \Phi+\Psi
$$

where we use the notation

$$
\Psi(\eta):=\sum_{x=1}^{L-1} g_{x}\left[1_{\left\{\eta_{x-1}=\eta_{x+1}=0\right\}}-(1-\delta) 1_{\left\{\eta_{x-1}=\eta_{x+1}=1\right\}}\right],
$$

with $\delta=2 /(1+\lambda)$. Setting

$$
\widetilde{\Psi}_{t}:=\Psi\left(\eta^{\wedge}(t)\right)-\Psi\left(\eta^{\vee}(t)\right),
$$

equation (4.2) becomes

$$
\frac{d}{d t} \mathbb{E} \widetilde{\Phi}_{t}=-\kappa_{L} \mathbb{E} \widetilde{\Phi}_{t}+\mathbb{E} \widetilde{\Psi}_{t}
$$

Therefore the claim (4.1) follows if we can prove that

$$
\mathbb{E} \widetilde{\Psi}_{t} \leqslant 0 .
$$

It will be convenient to rewrite $\mathbb{E} \widetilde{\Psi}_{t}$ as follows. Define

$$
\begin{aligned}
& \gamma_{0}(x, t)=\mathbb{P}\left(\eta_{x \pm 1}^{\vee}(t)=0\right)-\mathbb{P}\left(\eta_{x \pm 1}^{\wedge}(t)=0\right), \\
& \gamma_{1}(x, t)=\mathbb{P}\left(\eta_{x \pm 1}^{\vee}(t)=1\right)-\mathbb{P}\left(\eta_{x \pm 1}^{\wedge}(t)=1\right) .
\end{aligned}
$$

In this way,

$$
\mathbb{E} \widetilde{\Psi}_{t}=-\sum_{x=1}^{L-1} g_{x}\left[\gamma_{0}(x, t)-(1-\delta) \gamma_{1}(x, t)\right] .
$$

Clearly, by construction, $\gamma_{0}(x, t)=0$ for $x$ even and $\gamma_{1}(x, t)=0$ for $x$ odd. Note that $\gamma_{i}(x, t) \geqslant 0$ for all $t \geqslant 0$, all $x$ and $i=0,1$, by monotonicity (for instance, due to the constraint $\eta_{x} \geq 0$ and to 
monotonicity of the global coupling, $\eta_{x \pm 1}^{\vee}(t)=1$ whenever $\eta_{x \pm 1}^{\wedge}(t)=1$, and the non-negativity of $\gamma_{1}(x, t)$ immediately follows). In particular, this implies the estimate (4.6) if $\lambda \leqslant 1$, since in this case $\delta \geqslant 1$. The case $\lambda>1$ requires more work.

Define $a_{x}$ as the equilibrium probability that $\eta_{x-1}=\eta_{x+1}=0$ conditioned to the event that $\eta_{x}=\eta_{x+2}=1$; similarly, define $b_{x}$ as the equilibrium probability that $\eta_{x-1}=\eta_{x+1}=0$ conditioned on the event that $\eta_{x-2}=\eta_{x}=1$ :

$$
a_{x}=\mu^{+}\left[\eta_{x \pm 1}=0 \mid \eta_{x}=\eta_{x+2}=1\right], \quad b_{x}=\mu^{+}\left[\eta_{x \pm 1}=0 \mid \eta_{x-2}=\eta_{x}=1\right] .
$$

The proof of (4.6) in the case $\lambda>1$ is based on the next two results.

Lemma 4.1. For all $t \geqslant 0$, all $x=2, \ldots, L-2$ :

$$
\begin{aligned}
& \gamma_{0}(x-1, t) \geqslant a_{x-1} \gamma_{1}(x, t), \\
& \gamma_{0}(x+1, t) \geqslant b_{x+1} \gamma_{1}(x, t) .
\end{aligned}
$$

Lemma 4.2. Set

$$
\rho(x):=\min \left\{a_{x-1}, b_{x+1}\right\} .
$$

Then, uniformly in $L$ and $x=2, \ldots, L-2$ :

$$
\rho(x) \geqslant 1-\delta
$$

Once we have (4.9) and (4.10) we can estimate

$$
\sum_{x=2}^{L-2} g_{x} \gamma_{1}(x, t) \leqslant \frac{1}{2} \sum_{x=2}^{L-2} g_{x}\left\{a_{x-1}^{-1} \gamma_{0}(x-1, t)+b_{x+1}^{-1} \gamma_{0}(x+1, t)\right\} .
$$

Inserting in (4.7) and using (4.11) we arrive at

$$
-\mathbb{E} \widetilde{\Psi}(t) \geqslant \sum_{x=1}^{L-1}\left[g_{x}-\frac{g_{x-1}+g_{x+1}}{2}\right] \gamma_{0}(x, t) .
$$

Recalling that $\Delta g=-\kappa_{L} g$, the desired claim follows:

$$
-\mathbb{E} \widetilde{\Psi}(t) \geqslant \kappa_{L} \sum_{x=1}^{L-1} g_{x} \gamma_{0}(x, t) \geqslant 0 .
$$

\subsubsection{Proof of Lemma 4.1}

We first prove that for any odd $x=1, \ldots, L-3$

$$
\mathbb{P}\left(\eta_{x-1}^{\wedge}(t)=\eta_{x+1}^{\wedge}(t)=0\right) \leqslant a_{x} \mathbb{P}\left(\eta_{x}^{\wedge}(t)=\eta_{x+2}^{\wedge}(t)=1\right) .
$$

Let $A \subset \Omega_{L}^{+}$denote the subset of non-negative paths $\eta$ such that $\eta_{x-1}=\eta_{x+1}=0$. Also, let $B \subset \Omega_{L}^{+}$denote the subset of non-negative paths $\eta$ such that $\eta_{x}=\eta_{x+2}=1$. Note that $A \subset B$. If $\mu^{+}$denotes the equilibrium measure, we consider the conditional laws $\mu_{A}=\mu^{+}[\cdot \mid \eta \in A]$ and $\mu_{B}=\mu^{+}[\cdot \mid \eta \in B]$. It is not hard to show that we can find a coupling $\nu$ of $\left(\mu_{A}, \mu_{B}\right)$ such that 
$\nu\left(\eta_{A} \leqslant \eta_{B}\right)=1$ if $\eta_{A}$ is distributed according to $\mu_{A}$ and $\eta_{B}$ is distributed according to $\mu_{B}$. As discussed in Section 2.2.1 this can be obtained from the global coupling by letting time go to infinity. For any $\xi_{A} \in A$ and $\xi_{B} \in B$, we write $\nu\left(\xi_{B} \mid \xi_{A}\right)$ for the $\nu$-conditional probability of having $\eta_{B}=\xi_{B}$ given that $\eta_{A}=\xi_{A}$. We have $\nu\left(\xi_{B} \mid \xi_{A}\right)=0$ unless $\xi_{B} \geqslant \xi_{A}$.

Using the reversibility (2.28), the left hand side in (4.14) can be written as

$$
\sum_{\xi_{A} \in A} P_{t}\left(\wedge, \xi_{A}\right)=\sum_{\xi_{A} \in A} P_{t}\left(\xi_{A}, \wedge\right) \frac{\mu^{+}\left(\xi_{A}\right)}{\mu^{+}(\wedge)} .
$$

Note that for any $\xi_{A} \leqslant \xi_{B}$ monotonicity implies that $P_{t}\left(\xi_{A}, \wedge\right) \leqslant P_{t}\left(\xi_{B}, \wedge\right)$. Therefore we find

$$
\begin{aligned}
\sum_{\xi_{A} \in A} P_{t}\left(\xi_{A}, \wedge\right) \frac{\mu^{+}\left(\xi_{A}\right)}{\mu^{+}(\wedge)} & =\sum_{\xi_{A} \in A} \sum_{\xi_{B} \in B} \nu\left(\xi_{B} \mid \xi_{A}\right) P_{t}\left(\xi_{A}, \wedge\right) \frac{\mu^{+}\left(\xi_{A}\right)}{\mu^{+}(\wedge)} \\
& \leqslant \sum_{\xi_{A} \in A} \sum_{\xi_{B} \in B} \nu\left(\xi_{B} \mid \xi_{A}\right) P_{t}\left(\xi_{B}, \wedge\right) \frac{\mu^{+}\left(\xi_{A}\right)}{\mu^{+}(\wedge)} \\
& =\sum_{\xi_{A} \in A} \sum_{\xi_{B} \in B} \frac{\nu\left(\xi_{B}, \xi_{A}\right)}{\mu_{A}\left(\xi_{A}\right)} P_{t}\left(\wedge, \xi_{B}\right) \frac{\mu^{+}\left(\xi_{A}\right)}{\mu^{+}\left(\xi_{B}\right)}
\end{aligned}
$$

Clearly,

$$
\frac{\mu^{+}\left(\xi_{A}\right)}{\mu_{A}\left(\xi_{A}\right)}=\mu^{+}(A)
$$

and

$$
\sum_{\xi_{A} \in A} \nu\left(\xi_{B}, \xi_{A}\right)=\mu_{B}\left(\xi_{B}\right)=\frac{\mu^{+}\left(\xi_{B}\right)}{\mu^{+}(B)}
$$

Therefore

$$
\sum_{\xi_{A} \in A} \frac{\nu\left(\xi_{B}, \xi_{A}\right)}{\mu_{A}\left(\xi_{A}\right)} \frac{\mu^{+}\left(\xi_{A}\right)}{\mu^{+}\left(\xi_{B}\right)}=\frac{\mu^{+}(A)}{\mu^{+}(B)}=a_{x}
$$

This implies (4.14).

In a similar way one shows that for any odd $x=1, \ldots, L-3$

$$
\mathbb{P}\left(\eta_{x-1}^{\vee}(t)=\eta_{x+1}^{\vee}(t)=0\right) \geqslant a_{x} \mathbb{P}\left(\eta_{x}^{\vee}(t)=\eta_{x+2}^{\vee}(t)=1\right)
$$

The bounds (4.14) and (4.15) imply (4.9). The complementary bound (4.10) follows from the same arguments.

\subsubsection{Proof of Lemma 4.2}

We observe that, for $x$ even, $a_{x-1}=(1-\delta / 2) p_{x}$ where $1-\delta / 2=\lambda /(1+\lambda)$ is the equilibrium probability that $\eta_{x}=0$ given that $\eta_{x-1}=\eta_{x+1}=1$ and $p_{x}:=\mu_{x}^{+, \lambda}\left(\eta_{2}=0\right)$ is the equilibrium probability that $\eta_{2}=0$ in the system of length $x$. Similarly, $b_{x+1}=(1-\delta / 2) p_{L-x}$. In particular:

$$
\rho(x) \geqslant(1-\delta / 2) \min _{x \text { even }} p_{x}
$$


Therefore we need a bound of the form

$$
\min _{x \text { even }} p_{x} \geqslant \frac{1-\delta}{1-\delta / 2}
$$

Note that $\frac{1-\delta}{1-\delta / 2}=\frac{\lambda-1}{\lambda}$. We will show first that $p_{x}$ is non-increasing in $x$ and then that $p_{\infty}:=$ $\lim _{x \rightarrow \infty} p_{x} \geqslant(\lambda-1) / \lambda$.

Indeed, if $x<y$ and $x, y$ are both even, since the function $f=-1_{\eta_{2}=0}$ is increasing, the inequality $p_{x} \geqslant p_{y}$ is easily derived from the FKG inequality (2.27). Next, note that from (2.6)

$$
p_{\infty}=\lim _{x \rightarrow \infty} \mu_{x}^{\lambda / 2}\left(\eta_{2}=0\right)
$$

where $\mu_{x}^{\lambda}$ denotes the equilibrium measure without the wall in the system of length $x$ and with parameter $\lambda$. From [14, Th. 2.3] (or from Theorem 2.1 above) we have

$$
\lim _{x \rightarrow \infty} \mu_{x}^{\lambda}\left(\eta_{2}=0\right)=\lambda / 2 \exp (-2 F(\lambda))
$$

where $F$ is the free energy defined in Section 2.1. Since $F(\lambda)=0$ for $\lambda \leqslant 1$ we have $p_{\infty}=$ $\lambda / 4 \geqslant(\lambda-1) / \lambda$ for all $\lambda \leqslant 2$. As for $\lambda \geqslant 2$, one uses the explicit expression (2.12), which gives $p_{\infty}=(\lambda-1) / \lambda$. This ends the proof.

\subsection{Proof of (4.1) without the wall}

Here we assume $\lambda \geqslant 1$. In the model without the wall, we can repeat the computations leading to (4.5). The function $\Psi$ containing the correction terms from Lemma 2.3 is now given by

$$
\Psi(\eta):=(1-\delta) \sum_{x=1}^{L-1} g_{x}\left[1_{\left\{\eta_{x-1}=\eta_{x+1}=-1\right\}}-1_{\left\{\eta_{x-1}=\eta_{x+1}=1\right\}}\right],
$$

with $\delta=2 /(1+\lambda) \leqslant 1$. Setting again $\widetilde{\Psi}_{t}:=\Psi\left(\eta^{\wedge}(t)\right)-\Psi\left(\eta^{\vee}(t)\right)$, we arrive at the same expression given in (4.5). Therefore it suffices to show that $\mathbb{E} \widetilde{\Psi}_{t} \leqslant 0$. This in turn is an immediate consequence of the next lemma.

Lemma 4.3. For every even $x$ we have

$$
\begin{aligned}
& \mathbb{P}\left(\eta_{x \pm 1}^{\wedge}(t)=1\right) \geqslant \mathbb{P}\left(\eta_{x \pm 1}^{\wedge}(t)=-1\right), \\
& \mathbb{P}\left(\eta_{x \pm 1}^{\vee}(t)=-1\right) \geqslant \mathbb{P}\left(\eta_{x \pm 1}^{\vee}(t)=1\right) .
\end{aligned}
$$

Proof. By symmetry it suffices to prove (4.19) only. We use an argument similar to that of Lemma 4.1. Namely, call $A$ the set of all paths $\eta \in \Omega_{L}$ such that $\eta_{x \pm 1}=-1$ and $B$ the set of all paths $\eta \in \Omega_{L}$ such that $\eta_{x \pm 1}=1$ and let $\mu_{A}=\mu[\cdot \mid \eta \in A], \mu_{B}=\mu[\cdot \mid \eta \in B]$. Again, using the global coupling we construct a coupling $\nu$ of $\left(\mu_{A}, \mu_{B}\right)$ such that $\nu\left(\eta_{A} \leqslant \eta_{B}\right)=1$ if $\eta_{A}$ is distributed according to $\mu_{A}$ and $\eta_{B}$ according to $\mu_{B}$. We have $\nu\left(\xi_{B} \mid \xi_{A}\right)=0$ unless $\xi_{B} \geqslant \xi_{A}$. Using monotonicity we also have $P_{t}\left(\xi_{A}, \wedge\right) \leqslant P_{t}\left(\xi_{B}, \wedge\right)$ whenever $\xi_{B} \geqslant \xi_{A}$. Therefore the same computation as in the proof of Lemma 4.1 now gives

$$
\mathbb{P}\left(\eta_{x \pm 1}^{\wedge}(t)=-1\right)=\sum_{\xi_{A} \in A} P_{t}\left(\wedge, \xi_{A}\right) \leqslant \frac{\mu(A)}{\mu(B)} \sum_{\xi_{B} \in B} P_{t}\left(\wedge, \xi_{B}\right)=\mathbb{P}\left(\eta_{x \pm 1}^{\wedge}(t)=1\right),
$$

where we use the symmetry $\mu(A)=\mu(B)$. 


\section{$5 \quad$ Proof of Theorem 3.2 and related bounds}

\subsection{Upper bounds on the spectral gap}

We start with the proof of (3.5). Note that this bound can be derived from the independent estimate (3.6) by using (2.35). However, we show an explicit test function which reproduces the bound gap $\leqslant c / L$ in the localized phase $\left(i . e\right.$. when $\lambda>2$ for $\mathcal{L}^{+}$and when $\lambda>1$ for $\mathcal{L}$ ). The idea is reminiscent of an argument used in [4] for the low temperature Ising model.

Proposition 5.1. For every $\lambda>2$ there exists a constant $c \in(0, \infty)$ such that

$$
\frac{\mathcal{E}^{+}\left(f_{c}, f_{c}\right)}{\operatorname{Var}\left(f_{c}\right)} \leqslant \frac{32 c^{2}}{L}
$$

where for $a>0$

$$
f_{a}(\eta):=\exp \left(\frac{a}{L} \sum_{x=1}^{L-1} \eta_{x}\right) .
$$

The same holds without the wall, if $\lambda>1$.

Proof of Proposition 5.1. Let

$$
\psi(a):=\lim _{L \rightarrow \infty} \frac{1}{L} \log \mu_{L}^{+, \lambda}\left(f_{a}\right) .
$$

The limit exists since

$$
\log \mu_{L}^{+, \lambda}\left(f_{a}\right)=\log \sum_{\eta \in \Omega_{L}^{+}}\left(\lambda^{N(\eta)} f_{a}(\eta)\right)-\log Z_{L}^{+}(\lambda)
$$

and both terms in the right-hand side are super-additive in $L$ (both terms, once divided by $L$, tend to a finite limit for $L \rightarrow \infty$ : for the second one this follows from Theorem 2.1, while for the first one just note that $\left.\lambda^{N(\eta)} f_{a}(\eta) \leq \lambda^{L} e^{a L}\right)$. Observe also that $\psi(a)$ is non-decreasing in $a$ : this is obvious in presence of the wall since $\eta_{x} \geqslant 0$, and in absence of the wall this follows from the fact that $\psi(a)$ is convex and that

$$
\left.\partial_{a} \log \mu_{L}^{\lambda}\left(f_{a}\right)\right|_{a=0}=0 .
$$

We will show that there exists $\bar{a} \in(0, \infty)$ such that $\psi(a)=0$ for $a \leqslant \bar{a}$ and $\psi(a)>0$ for $a>\bar{a}$. Then, choosing $\bar{a} / 2<c<\bar{a}$, one has that, for $L$ sufficiently large,

$$
\operatorname{Var}\left(f_{c}\right) \geqslant(1 / 2) \mu_{L}^{+, \lambda}\left(f_{c}^{2}\right)
$$

As for the Dirichlet form, since

$$
\left|Q_{x}^{+} f_{c}(\eta)-f_{c}(\eta)\right| \leqslant \frac{4 c}{L} f_{c}(\eta),
$$

one deduces easily from $(2.29)$ that

$$
\mathcal{E}^{+}\left(f_{c}, f_{c}\right) \leqslant \frac{16 c^{2}}{L} \mu_{L}^{+, \lambda}\left(f_{c}^{2}\right) .
$$


The statement of the proposition is then proven once we show the existence of the $\bar{a}$ introduced above, and it is here that the assumption $\lambda>2$ will play a role. Note that $\bar{a}$ is uniquely defined by the monotonicity of $\psi(a)$, so we have only to show that it is neither zero nor infinity. First of all,

$$
\mu_{L}^{+, \lambda}\left(f_{a}\right) \geqslant \mu_{L}^{+, \lambda}\left(f_{a} 1_{\eta=\wedge}\right) \geqslant e^{(a / 8) L}
$$

if $a$ is sufficiently large, so that $\bar{a}<\infty$. Conversely, from Jensen's inequality

$$
f_{a} \leqslant \frac{1}{L} \sum_{i=1}^{L} e^{a \eta_{x}}
$$

and from Lemma 2.2 we see that $\mu_{L}^{+, \lambda}\left(f_{a}\right) \leqslant C$ for some $C$ independent of $L$ if, say, $a<F(\lambda) / 2$. In this case $\psi(a)=0$ and therefore $\bar{a}>0$.

The proof of the Proposition in the case of $\lambda>1$ and no wall is essentially identical.

In the delocalized phase and at the critical point, on the other hand, we can do better and show a test function which gives the (optimal) behavior of order $L^{-2}$. The next result proves Eq. (3.3).

Proposition 5.2. Let

$$
f(\eta):=\sum_{x=1}^{L} \sin \left(\frac{\pi(x-1 / 2)}{L}\right)\left(\eta_{x}-\eta_{x-1}\right) .
$$

There exists $c<\infty$ such that for every $\lambda \leqslant 2$ the following holds:

$$
\frac{\mathcal{E}^{+}(f, f)}{\operatorname{Var}(f)} \leqslant \frac{c}{L^{2}}
$$

Proof. Set for convenience $\phi_{x}:=\eta_{x}-\eta_{x-1}$ and $h_{L}(x):=\sin (\pi(x-1 / 2) / L)$. It is clear from the symmetry $x \leftrightarrow(L-x)$ that $\mu_{L}^{+, \lambda}(f)=0$. The Dirichlet form is easily estimated from above: since the occurrence of a flip at site $x$ has the effect of exchanging the values of $\phi_{x}$ and $\phi_{x+1}$, one has

$$
\mathcal{E}^{+}(f, f) \leqslant \sum_{x=1}^{L} \mu_{L}^{+, \lambda}\left(\phi_{x} \neq \phi_{x-1}\right)\left[h_{L}(x+1)-h_{L}(x)\right]^{2} .
$$

Therefore, for $L$ sufficiently large, one has

$$
\mathcal{E}^{+}(f, f) \leqslant \frac{c}{L} \int_{0}^{1} \cos (\pi s)^{2} d s
$$

As for the second moment of $f$, what we need to show is that

$$
\mu_{L}^{+, \lambda}\left(f^{2}\right) \geqslant c L
$$

with $c \in(0, \infty)$. 
To this end we note that

$$
\begin{aligned}
L^{-1 / 2} f(\eta) & =\frac{1}{\sqrt{L}} \sum_{x=1}^{L-1} \eta_{x}\left(h_{L}(x)-h_{L}(x+1)\right) \\
& =-\frac{\pi}{L^{3 / 2}} \sum_{x=1}^{L-1} \eta_{x} \cos \left(\frac{\pi(x-1 / 2)}{L}\right)+O\left(L^{-1 / 2}\right)
\end{aligned}
$$

where the estimate on the error term is uniform in $\eta$. Introducing the continuous, piecewise linear process $\left\{\eta_{s}^{(L)}\right\}_{s \in[0,1]}$ such that $\eta_{x / L}^{(L)}:=L^{-1 / 2} \eta_{x}$ for $x=0, \ldots, L$ and $\partial_{s}^{2} \eta_{s}^{(L)}=0$ for $s \in(x / L,(x+1) / L)$, one has

$$
L^{-1 / 2} f(\eta)=-\pi \int_{0}^{1} d s \eta_{s}^{(L)} \cos (\pi s)+O\left(L^{-1 / 2}\right) .
$$

It is easy to see that for every $k>0$

$$
\sup _{L} \sup _{x \in \Lambda} \mu_{L}^{+, \lambda}\left(\frac{\left(\eta_{x}\right)^{k}}{L^{k / 2}}\right)<\infty
$$

(Indeed, using monotonicity a couple of times,

$$
\mu_{L}^{+, \lambda}\left(\frac{\left(\eta_{x}\right)^{k}}{L^{k / 2}}\right) \leqslant \mathbf{E}\left(\frac{\left(\eta_{L}\right)^{k}}{L^{k / 2}} \mid \eta_{y}>0, \quad \forall 1 \leqslant y \leqslant L\right)
$$

and the latter expression is seen to be finite uniformly in $L$ using the "Ballot Theorem" [8, Sec. III.1] plus Stirling's formula.) Equation (5.12) is therefore proven if we show that

$$
\int_{0}^{1} d t \int_{0}^{1} d s \cos (\pi t) \cos (\pi s) \mu_{L}^{+, \lambda}\left(\eta_{t}^{(L)} \eta_{s}^{(L)}\right) \stackrel{L \rightarrow \infty}{\rightarrow} c \in(0, \infty) .
$$

Consider first the case $\lambda<2$, in which case

$$
\mu_{L}^{+, \lambda}\left(\eta_{t}^{(L)} \eta_{s}^{(L)}\right) \stackrel{L \rightarrow \infty}{\rightarrow} \mu_{X}\left(X_{t} X_{s}\right)
$$

where $X$. is the Brownian bridge in $[0,1]$ conditioned to be non-negative (i.e., the Bessel bridge of dimension 3 from 0 to 0 ), whose law we denote by $\mu_{X}(\cdot)$. Equation (5.17) follows from the convergence in distribution of the process $\eta^{\left({ }^{L}\right)}$ to $X$. (this is proven in [7] in a slightly different setting, but the techniques developed there can be extended to our case; see also [16]), together with the uniform integrability (5.15). Thanks to (5.15) we can take the $L \rightarrow \infty$ limit inside the integral in (5.16) and the limit is

$$
\operatorname{Var}_{\mu_{X}}\left(\int_{0}^{1} d s \cos (\pi s) X_{s}\right)>0
$$

Together with (5.11) this concludes the proof of the proposition in the strictly delocalized case. It remains to consider the critical case $\lambda=2$. In this case, [5, Th. 5.1] plus (5.15) imply that

$$
\mu_{L}^{+, 2}\left(\eta_{t}^{(L)} \eta_{s}^{(L)}\right) \stackrel{L \rightarrow \infty}{\rightarrow} \mu_{B}\left(\left|B_{t}\right|\left|B_{s}\right|\right)
$$

$B$. being the Brownian bridge on $[0,1]$ with law $\mu_{B}$. Therefore, one finds in this case

$$
\mu_{L}^{+, 2}\left(f^{2} / L\right) \stackrel{L \rightarrow \infty}{\longrightarrow} \pi^{2} \operatorname{Var}_{\mu_{B}}\left(\int_{0}^{1} d s \cos (\pi s)\left|B_{s}\right|\right)>0
$$

and the conclusion follows as before. 


\subsection{Lower bounds on mixing times}

We begin with the proof of (3.4) for the system with the wall at $0<\lambda<2$.

Proposition 5.3. For any $\lambda<2$,

$$
T_{\text {mix }} \geqslant\left(\frac{1}{2 \pi^{2}}+o(1)\right) L^{2} \log L .
$$

Let $\Phi$ and $\Psi$ be the functions defined in (2.39) and (4.4) respectively. We consider their evolutions when the process starts in the maximal configuration $\wedge$ and set $\hat{\Phi}_{t}:=\Phi\left(\eta^{\wedge}(t)\right)$ and $\hat{\Psi}_{t}:=$ $\Psi\left(\eta^{\wedge}(t)\right)$. From the computation in Lemma 2.3 , as in (4.3) we obtain

$$
\frac{d}{d t} \mathbb{E}\left[\hat{\Phi}_{t}\right]=P_{t} \mathcal{L} \Phi(\wedge)=-\kappa_{L} \mathbb{E}\left[\hat{\Phi}_{t}\right]+\mathbb{E}\left[\hat{\Psi}_{t}\right]
$$

where as usual we omit the + superscript and write $\mathcal{L}$ for $\mathcal{L}^{+}$and $P_{t}$ for $P_{t}^{+}$. Next, we claim that for any $\lambda<2$, there exists $c(\lambda)<\infty$ such that for all $t \geqslant 0, L \geqslant 2$ :

$$
\mathbb{E}\left[\hat{\Psi}_{t}\right] \geqslant-c(\lambda) L^{-1 / 2} .
$$

To prove this, observe that

$$
\mathbb{E}\left[\hat{\Psi}_{t}\right] \geqslant-(1-\delta) \sum_{x=1}^{L-1} g_{x} \mathbb{P}\left(\eta_{x \pm 1}^{\wedge}(t)=1\right) .
$$

Note that if $\lambda \leqslant 1$ then $\delta \geqslant 1$ and therefore $\mathbb{E}\left[\hat{\Psi}_{t}\right] \geqslant 0$. If $\lambda \in(1,2)$ we use the following argument to prove (5.22). Monotonicity allows us to bound $\mathbb{P}\left(\eta_{x \pm 1}^{\wedge}(t)=1\right)$ from above by the equilibrium probability $\mu^{+}\left(\eta_{x \pm 1}=1\right)$. The latter, in turn, for each $2 \leqslant x \leqslant L-2$ and $\lambda<2$ is estimated with

$$
\mu^{+}\left(\eta_{x \pm 1}=1\right)=\frac{1+\lambda}{\lambda} \mu^{+}\left(\eta_{x}=0\right) \leqslant c(\lambda) \frac{L^{3 / 2}}{(L-x)^{3 / 2} x^{3 / 2}},
$$

where $c(\lambda)$ is a suitable constant. Note that (5.23) follows from (2.13) using

$$
\mu^{+}\left(\eta_{x}=0\right)=\frac{Z_{x}^{+}(\lambda) Z_{L-x}^{+}(\lambda)}{Z_{L}^{+}(\lambda)}=\frac{1}{2} \frac{Z_{x}(\lambda / 2) Z_{L-x}(\lambda / 2)}{Z_{L}(\lambda / 2)}
$$

and the fact that $\lambda<2$. Once we have (5.23), using $g_{x} \leqslant \frac{\pi x}{L}$ for $x \leqslant L / 2$ and $g_{x} \leqslant \frac{\pi(L-x)}{L}$ for $x \geqslant L / 2$ we obtain

$$
\mathbb{E}\left[\hat{\Psi}_{t}\right] \geqslant-c(\lambda) \sum_{x=1}^{L / 2} \frac{1}{x^{1 / 2} L},
$$

with a new constant $c(\lambda)$. This implies the claim (5.22).

Next, we integrate (5.21) using (5.22) to obtain

$$
\begin{aligned}
\mathbb{E}\left[\hat{\Phi}_{t}\right] & \geqslant e^{-\kappa_{L} t} \hat{\Phi}_{0}-c(\lambda) L^{-1 / 2} \int_{0}^{t} e^{-\kappa_{L}(t-s)} d s \\
& \geqslant e^{-\kappa_{L} t} \hat{\Phi}_{0}-c(\lambda) L^{-1 / 2} \kappa_{L}^{-1} .
\end{aligned}
$$


Therefore we have shown that for each $\lambda<2$, for some constant $c(\lambda)$, for all $t \geqslant 0$ and $L \geqslant 2$ :

$$
\mathbb{E}\left[\hat{\Phi}_{t}\right] \geqslant e^{-\kappa_{L} t} \hat{\Phi}_{0}-c(\lambda) L^{3 / 2} .
$$

Since $\hat{\Phi}_{0} \geqslant c L^{2}$ and $\kappa_{L} \sim \pi^{2} / 2 L^{2}$, from (5.24) we see that $\mathbb{E}\left[\hat{\Phi}_{t}\right]$ is much larger than its equilibrium value $\mathbb{E}\left[\hat{\Phi}_{\infty}\right]=O\left(L^{3 / 2}\right)$ for times $t$ within, say, $\frac{1}{2 \pi^{2}} L^{2} \log L$. However, this is still not enough to prove that the mixing time is at least of order $L^{2} \log L$, since the $L_{\infty}$ norm of $\Phi$ is of order $L^{2}$.

Following Wilson [23], we turn to an estimate on the variance of $\hat{\Phi}_{t}$.

Lemma 5.4. For every $\lambda<2$ there exists $c(\lambda)<\infty$ such that for all $t>0$ :

$$
\operatorname{Var}\left(\hat{\Phi}_{t}\right)=\mathbb{E}\left[\hat{\Phi}_{t}^{2}\right]-\mathbb{E}\left[\hat{\Phi}_{t}\right]^{2} \leqslant c(\lambda) L^{7 / 2}
$$

Proof. We start by giving an upper bound on $\mathbb{E}\left[\hat{\Phi}_{t}^{2}\right]$. Recall from $(2.25)$ that

$$
\mathcal{L} \Phi^{2}=\sum_{x=1}^{L-1}\left[Q_{x} \Phi^{2}-\Phi^{2}\right]
$$

where $Q_{x}$ is the equilibrium measure at $x$ conditioned on the configuration $\eta$ outside of $x$. Writing $Q_{x}(\xi \mid \eta):=\mu^{+}\left[\xi \mid \eta_{y}, y \neq x\right]$ for the associated kernel, for every $\eta \in \Omega_{L}^{+}$we have

$$
\begin{aligned}
Q_{x} \Phi^{2}(\eta) & =\sum_{\xi} Q_{x}(\xi \mid \eta) \Phi^{2}(\xi) \\
& =\sum_{\xi} Q_{x}(\xi \mid \eta)\left[\Phi^{2}(\eta)+2 \Phi(\eta)(\Phi(\xi)-\Phi(\eta))+(\Phi(\xi)-\Phi(\eta))^{2}\right]
\end{aligned}
$$

We can estimate $\sum_{\xi} Q_{x}(\xi \mid \eta)(\Phi(\xi)-\Phi(\eta))^{2} \leqslant 4$ for each $x$ and $\eta$. Indeed, each transition can at most change the function $\Phi$ by 2 . Therefore

$$
\mathcal{L} \Phi^{2}(\eta) \leqslant 4 L+2 \Phi(\eta) \mathcal{L} \Phi(\eta)=4 L-2 \kappa_{L} \Phi(\eta)^{2}+2 \Phi(\eta) \Psi(\eta)
$$

where we have used again (4.3) in the last step. In conclusion, inserting (5.26) in the identity

$$
\frac{d}{d t} \mathbb{E}\left[\hat{\Phi}_{t}^{2}\right]=P_{t} \mathcal{L} \Phi^{2}(\wedge)
$$

and integrating we obtain

$$
\mathbb{E}\left[\hat{\Phi}_{t}^{2}\right] \leqslant e^{-2 \kappa_{L} t}\left(\hat{\Phi}_{0}\right)^{2}+4 L \kappa_{L}^{-1}+2 \int_{0}^{t} \mathbb{E}\left[\hat{\Phi}_{s} \hat{\Psi}_{s}\right] e^{-\kappa_{L}(t-s)} d s .
$$

To estimate the last term in (5.27) we note that $\hat{\Phi}_{s} \leqslant \hat{\Phi}_{0}=O\left(L^{2}\right)$ uniformly. Moreover, for any $\lambda>0, s \geqslant 0$ :

$$
\begin{aligned}
\mathbb{E}\left[\hat{\Psi}_{s}\right] & \leqslant \sum_{x=1}^{L-1} g_{x}\left[\mathbb{P}\left(\eta_{x \pm 1}^{\wedge}(s)=0\right)+\mathbb{P}\left(\eta_{x \pm 1}^{\wedge}(s)=1\right)\right] \\
& \leqslant \sum_{x=1}^{L-1} g_{x}\left[\mu^{+}\left(\eta_{x \pm 1}=0\right)+\mu^{+}\left(\eta_{x \pm 1}=1\right)\right]
\end{aligned}
$$


where the last step follows from monotonicity. As in (5.23) we have the following equilibrium bounds valid for any $\lambda<2$ :

$$
\mu^{+}\left(\eta_{x \pm 1}=i\right) \leqslant c(\lambda) \frac{L^{3 / 2}}{(L-x)^{3 / 2} x^{3 / 2}}, \quad i=0,1 .
$$

As in the proof of (5.22) these estimates imply

$$
\mathbb{E}\left[\hat{\Psi}_{s}\right] \leqslant c(\lambda) L^{-1 / 2} .
$$

From (5.27) we therefore obtain

$$
\begin{aligned}
\mathbb{E}\left[\hat{\Phi}_{t}^{2}\right] & \leqslant e^{-2 \kappa_{L} t} \hat{\Phi}_{0}^{2}+4 L \kappa_{L}^{-1}+c(\lambda) \kappa_{L}^{-1} \hat{\Phi}_{0} L^{-1 / 2} \\
& \leqslant e^{-2 \kappa_{L} t} \hat{\Phi}_{0}^{2}+c(\lambda) L^{7 / 2} .
\end{aligned}
$$

From (5.24) we know that $\mathbb{E}\left[\hat{\Phi}_{t}\right]^{2} \geqslant e^{-2 \kappa_{L} t} \hat{\Phi}_{0}^{2}-c(\lambda) L^{7 / 2}$ so that we deduce the upper bound (5.25).

Using Lemma 5.4 we can finish the proof of Proposition 5.3. Letting $t \rightarrow \infty$ in (5.25) we obtain a bound on the equilibrium variance

$$
\operatorname{Var}_{\mu^{+}}(\Phi)=\operatorname{Var}\left(\hat{\Phi}_{\infty}\right) \leqslant c(\lambda) L^{7 / 2}
$$

Define the set

$$
A_{\gamma}=\left\{\eta: \Phi(\eta) \leqslant L^{2-\gamma}\right\}, \quad \gamma \in(0,1 / 4) .
$$

Since $\mu^{+}(\Phi) \leqslant \sum_{x} \mu^{+}\left(\eta_{x}\right) \leqslant c L^{3 / 2}$ we see that, from Chebyshev's inequality and (5.31):

$$
\begin{aligned}
1-\mu^{+}\left(A_{\gamma}\right) & \leqslant \mu^{+}\left(\left|\Phi-\mu^{+}(\Phi)\right| \geqslant \frac{1}{2} L^{2-\gamma}\right) \\
& \leqslant 4 L^{-4+2 \gamma} \operatorname{Var}_{\mu^{+}}(\Phi) \leqslant c(\lambda) L^{-\frac{1}{2}+2 \gamma} .
\end{aligned}
$$

Let $P_{t}(\wedge, \cdot)$ denote the distribution of $\eta^{\wedge}(t)$. Using (5.24) we see that if $t \kappa_{L} \leqslant a \log L$ for some $a<\gamma$ then $\hat{\Phi}_{t} \leqslant L^{2-\gamma}$ implies $\left|\hat{\Phi}_{t}-\mathbb{E}\left(\hat{\Phi}_{t}\right)\right| \geqslant c L^{2-a}$, for some $c>0$, for all $L$ large enough. From Chebyshev's inequality and (5.25) we then have

$$
\begin{aligned}
P_{t}\left(\wedge, A_{\gamma}\right) & \leqslant \mathbb{P}\left(\left|\hat{\Phi}_{t}-\mathbb{E}\left(\hat{\Phi}_{t}\right)\right| \geqslant c L^{2-a}\right) \\
& \leqslant c^{-2} L^{-4+2 a} \operatorname{Var}\left(\hat{\Phi}_{t}\right) \leqslant c(\lambda) L^{-\frac{1}{2}+2 a} .
\end{aligned}
$$

In conclusion, taking $\gamma=\left(\frac{1}{4}-\varepsilon\right), a=\gamma-\varepsilon$ we see that for $L$ sufficiently large we have

$$
\left\|P_{t}(\wedge, \cdot)-\mu^{+}\right\|_{\mathrm{var}} \geqslant\left|P_{t}\left(\wedge, A_{\gamma}\right)-\mu^{+}\left(A_{\gamma}\right)\right| \geqslant 1-L^{-\varepsilon},
$$

whenever $t \leqslant\left(\frac{1}{4}-2 \varepsilon\right) \kappa_{L}^{-1} \log L$. Since $\kappa_{L} \sim \pi^{2} / 2 L^{2}$, this ends the proof of Proposition 5.3 . 


\subsection{A universal lower bound on the mixing time}

Here we shall prove the bound (3.6) and the corresponding estimate in Theorem 3.5.

Theorem 5.5. Both with and without the wall, for every $\lambda>0$ :

$$
T_{\text {mix }} \geqslant L^{2} / 32 \text {. }
$$

The proof is divided in three steps. First we prove the statement at $\lambda=\infty$ for the system with the wall. Then we extend it to any $\lambda>0$ with the wall and finally we show how to prove it for all $\lambda>0$ without the wall.

\subsection{1 $\lambda=\infty$ with the wall}

Recall that here $\mu^{+}=\delta_{\vee}$ is the Dirac mass at the minimal configuration $\vee$. In this situation, the definitions of generator, spectral gap and Dirichlet form as given in Section 2.2 do not make sense, and the dynamics is defined as in the introduction, with $\lambda /(1+\lambda)$ replaced by 1 . In other words, the rules for updating $\eta_{x}$ become:

- if $\eta_{x-1} \neq \eta_{x+1}$, do nothing;

- if $\eta_{x-1}=\eta_{x+1}=j$ and $j \neq 1$, set $\eta_{x}=j \pm 1$ with equal probabilities;

- if $\eta_{x-1}=\eta_{x+1}=1$, set $\eta_{x}=0$ with probability 1 .

Similar considerations hold for the $\lambda=\infty$ dynamics without the wall.

We want to estimate the expected time needed to go from $\wedge$ to $\vee$. Following a well known argument using the mapping with simple exclusion (see e.g. the proof of Theorem 1.3 in [11]) we would obtain a bound of the form $L^{2} / \log L$. We shall remove the spurious $\log L$ factor by means of the following argument.

We suppose for simplicity that $L / 2$ is even (it is straightforward to modify the construction in the case $L / 2$ odd). Let $D$ denote the square identified by the four vertices with coordinates $d_{1}=\left(\frac{L}{2}, \frac{L}{2}\right), d_{2}=\left(\frac{L}{4}, \frac{L}{4}\right), d_{3}=\left(\frac{3 L}{4}, \frac{L}{4}\right)$ and $d_{4}=\left(\frac{L}{2}, 0\right)$. Given a path $\eta \in \Omega_{L}^{+}$we call $q_{1}(\eta)$ and $q_{2}(\eta)$ the points where $\eta$ crosses the lines $d_{2}-d_{4}$ and $d_{3}-d_{4}$, respectively, with the rule that if the path $\eta$ touches the line in more than one point we use the lowest, i.e. the closest to $d_{4}$, see Figure 3. We call $\eta_{D}$ the portion of the path $\eta$ between $q_{1}(\eta)$ and $q_{2}(\eta)$. Also, we need to introduce the map which associates each path $\eta$ with the minimal path compatible with the portion $\eta_{D}$, see the dashed lines in Figure 3. We write $\mathfrak{T}_{D} \eta$ for this new configuration. Note that $\mathfrak{T}_{D} \eta \leqslant \eta$ for every $\eta$. If $\eta$ is such that $q_{1}(\eta)=q_{2}(\eta)=d_{4}$, i.e. if $\eta_{\frac{L}{2}}=0$, then $\mathfrak{T}_{D} \eta=\vee$. Also, $\mathfrak{T}_{D} \wedge=\wedge$.

Let $B_{D}(\eta)$ denote the area of the region inside the square $D$ enclosed by the path $\eta_{D}$ and the broken line joining points $q_{1}(\eta), d_{4}, q_{2}(\eta)$. The area $B_{D}$ will be measured by the number of elementary $\sqrt{2} \times \sqrt{2}$ squares it contains, so that e.g. $B_{D}(\wedge)=L^{2} / 16$.

Let $\eta(t)=\eta^{\wedge}(t)$ denote the time evolution starting from $\eta(0)=\wedge$. We shall consider a modified evolution $\xi(t)$ that can be coupled to $\eta(t)$ in such a way that $\xi(t) \leqslant \eta(t)$. We use the same Poisson clocks and the same "coins" (see Section 2.2.1) for the two processes. When a clock rings we 


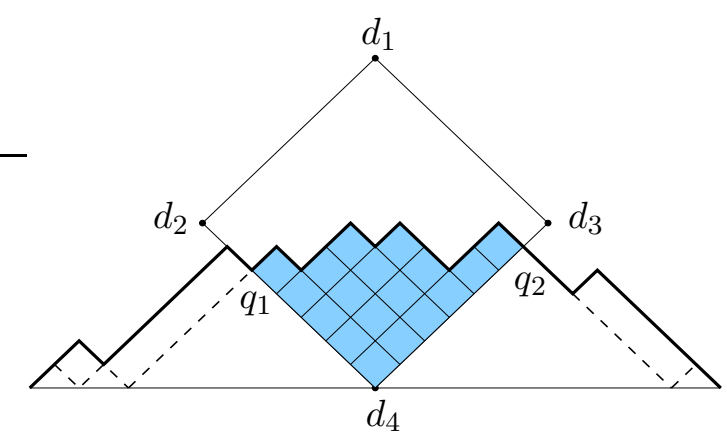

Figure 3: The square $D$ and the area $B_{D}(\eta)$ for a given path $\eta \in \Omega_{L}^{+}$.

update as in the global coupling with $(\eta, \xi) \rightarrow\left(\eta^{\prime}, \xi^{\prime}\right)$. After this updating the configuration $\xi^{\prime}$ is replaced by $\mathfrak{T}_{D} \xi^{\prime}$. The net result is therefore the updating $(\eta, \xi) \rightarrow\left(\eta^{\prime}, \mathfrak{T}_{D} \xi^{\prime}\right)$ where $(\eta, \xi) \rightarrow\left(\eta^{\prime}, \xi^{\prime}\right)$ is a standard update under the global coupling. Since $\mathfrak{T}_{D} \xi \leqslant \xi$ we have $\eta(t) \geqslant \xi(t)$ almost surely.

We set $\xi(0)=\eta(0)=\wedge$. We want to estimate the expected value of $B_{D}(\xi(t))$ from below. Observe that $\xi(t)$ lives in the space $\widetilde{\Omega}=\left\{\sigma \in \Omega_{L}^{+}: \mathfrak{T}_{D} \sigma=\sigma\right\}$. If $\mathcal{G}$ stands for the generator of the Markov chain $\xi(t)$, then we claim that, for any $\xi \in \widetilde{\Omega}$

$$
\mathcal{G} B_{D}(\xi) \geqslant-1
$$

To prove (5.34) note that $B_{D}(\xi)$ can only change by \pm 1 according to whether there is a mountain/valley in the path $\xi_{D}$. Each valley in $\xi_{D}$ contributes with $\frac{1}{2}$ to (5.34), if $\xi \neq \vee$. Moreover, each mountain in $\xi_{D}$ contributes with $-\frac{1}{2}$ to (5.34) unless reversing it would result in the configuration $\vee$, in which case its contribution to (5.34) is -1 . However the number of mountains minus the number of valleys in $\xi_{D}$ is always 1 (unless $\xi=\vee$, in which case $\mathcal{G} B_{D}(\xi)=0$ ). This implies (5.34).

From (5.34) we know that the martingale

$$
M_{t}=B_{D}(\xi(t))-B_{D}(\xi(0))-\int_{0}^{t} \mathcal{G} B_{D}(\xi(s)) d s,
$$

satisfies $0=\mathbb{E} M_{t} \leqslant \mathbb{E} B_{D}(\xi(t))-B_{D}(\xi(0))+t$ or,

$$
\mathbb{E} B_{D}(\xi(t)) \geqslant B_{D}(\wedge)-t .
$$

Setting $f(\eta):=B_{D}(\eta) / B_{D}(\wedge)$, we have $\mathbb{E} f(\eta(t)) \geqslant \mathbb{E} B_{D}(\xi(t)) / B_{D}(\wedge)$ and $\delta_{\vee}(f)=0$. Moreover, $\|f\|_{\infty} \leqslant 1$ and therefore

$$
\begin{aligned}
\left\|P_{t}(\wedge, \cdot)-\delta_{\vee}\right\|_{\text {var }} & \geqslant\left|P_{t}(\wedge, \cdot)(f)-\delta_{\vee}(f)\right| \\
& =\mathbb{E} f(\eta(t)) \geqslant 1-\frac{t}{B_{D}(\wedge)} .
\end{aligned}
$$

Since $B_{D}(\wedge)=\frac{L^{2}}{16}$ we have $T_{\text {mix }} \geqslant(e-1) L^{2} / 16 e \geqslant L^{2} / 32$. 


\subsection{2 $\lambda>0$ with the wall}

Let $\eta(t)$ denote the evolution with the wall, for a given $\lambda>0$, and with maximal initial condition $\eta(0)=\wedge$. If $\xi(t)$ denotes the process defined above, with $\xi(0)=\wedge$, we can couple the two processes in such a way that $\eta(t) \geqslant \xi(t)$ almost surely and therefore $\mathbb{E} B_{D}(\eta(t)) \geqslant \mathbb{E} B_{D}(\xi(t)) \geqslant B_{D}(\wedge)-t$ by (5.35). Set again $f(\eta):=B_{D}(\eta) / B_{D}(\wedge)$. Since $\mu^{+}\left(B_{D}\right) \leqslant \mu^{+}(A)=O\left(L^{3 / 2}\right)$ uniformly in $\lambda>0$, the equilibrium average of $f$ satisfies $\mu^{+}(f)=O\left(L^{-1 / 2}\right)$. Therefore

$$
\begin{aligned}
\left\|P_{t}(\wedge, \cdot)-\mu^{+}\right\|_{\text {var }} & \geqslant\left|P_{t}(\wedge, \cdot)(f)-\mu^{+}(f)\right| \\
& =\mathbb{E} f(\eta(t))+O\left(L^{-1 / 2}\right) \geqslant 1-\frac{t}{B_{D}(\wedge)}+O\left(L^{-1 / 2}\right) .
\end{aligned}
$$

As in (5.36) we obtain $T_{\text {mix }} \geqslant L^{2} / 32$ provided $L$ is sufficiently large.

\subsection{3 $\lambda>0$ without the wall}

Call $\eta(t)$ the evolution without the wall, for a given $\lambda>0$, and with maximal initial condition $\eta(0)=\wedge$. We can use the same arguments given above but we have to modify the process $\xi$ in order to satisfy the monotonicity $\eta(t) \geqslant \xi(t)$. Recall the construction of the square $D$ and the associated path $\eta_{D}$, see Figure 3 . The transformation $\mathfrak{T}_{D}$ here will be defined as follows. Given the portion of the path $\eta_{D}$ then $\mathfrak{T}_{D} \eta$ is the minimal configuration $\eta^{\prime} \in \Omega_{L}$ (i.e. without the wall) such that $\eta_{D}^{\prime}=\eta_{D}$. Also, we add the rule that if $\eta_{\frac{L}{2}} \leqslant 0$ then $\mathfrak{T}_{D} \eta=\vee=-\wedge$. In this way, if the process $\xi(t)$ is defined as before (but with the new $\mathfrak{T}_{D}$ ), then we can guarantee the domination $\eta(t) \geqslant \xi(t)$. In particular, $B_{D}(\eta(t)) \geqslant B_{D}(\xi(t))$. Note that, by the same arguments, our process $\xi(t)$ satisfies (5.34) and therefore (5.35). Then we set $f(\eta):=B_{D}(\eta) / B_{D}(\wedge)$ and observe that the equilibrium average of $f$ satisfies $\mu(f) \leqslant \mu^{+}(f)=O\left(L^{-1 / 2}\right)$ uniformly in $\lambda>0$. The rest of the argument is the same as for (5.37).

\subsection{On the mixing time at $\lambda=\infty$}

The next result is an upper bound on the mixing time at $\lambda=\infty$, showing that the estimate of Theorem 5.5 is sharp up to constant factors in this case.

Proposition 5.6. For $\lambda=\infty$, both with and without the wall

$$
T_{\text {mix }} \leqslant L^{2}
$$

Proof. We first give the proof for the system with the wall. Let $A(\eta)$ denote the area under the path $\eta$ :

$$
A(\eta):=\sum_{x \in \Lambda} \eta_{x}
$$

Then, $A\left(\eta^{\wedge}(t)\right)$ is a process on $\{A(\vee), \ldots, A(\wedge)\}$, where $A(\wedge)=\frac{L^{2}}{4}$ is the maximal value and $A(\vee)=\frac{L}{2}$ is the minimal value. The process starts at $A(\wedge)$, has \pm 2 increments and is killed 
upon hitting $A(\mathrm{~V})$. We want an upper bound on the expected value of $\tau$, where $\tau$ denotes the hitting time of $A(\mathrm{~V})$. It will be shown below that

$$
\mathcal{L} A(\eta) \leqslant-1, \quad \forall \eta \neq \vee .
$$

Assume (5.40) and consider the martingale

$$
M_{t}=A\left(\eta_{t}\right)-A(\wedge)-\int_{0}^{t} \mathcal{L} A\left(\eta_{s}\right) d s,
$$

where $\eta_{t}:=\eta^{\wedge}(t)$. By the optional stopping theorem and (5.40) we obtain

$$
0=\mathbb{E} M_{\tau}=\mathbb{E} A\left(\eta_{\tau}\right)-A(\wedge)-\mathbb{E} \int_{0}^{\tau} \mathcal{L} A\left(\eta_{s}\right) d s \geqslant A(\vee)-A(\wedge)+\mathbb{E} \tau
$$

This implies $\mathbb{E} \tau \leqslant A(\wedge)-A(\vee)$ and therefore, using (2.33) and Markov's inequality:

$$
\begin{aligned}
\left\|P_{t}(\wedge, \cdot)-\delta_{\vee}\right\|_{\mathrm{var}} & \leqslant \mathbb{P}(\tau>t) \\
& \leqslant \frac{1}{t}(A(\wedge)-A(\vee)) .
\end{aligned}
$$

This gives the mixing time bound $T_{\text {mix }} \leqslant e(A(\wedge)-A(\vee)) \leqslant \frac{e}{4} L^{2} \leqslant L^{2}$.

It remains to prove (5.40). From Lemma 2.3 , with $\delta=0$, we have

$$
\mathcal{L} A(\eta)=\sum_{x}(\Delta \eta)_{x}+\sum_{x=1}^{L-1}\left[1_{\left\{\eta_{x-1}=\eta_{x+1}=0\right\}}-1_{\left\{\eta_{x-1}=\eta_{x+1}=1\right\}}\right] .
$$

Note that for any $\eta \in \Omega_{L}^{+}$we have $\sum_{x}(\Delta \eta)_{x}=-1$ (for a non-negative path the number of mountains exceeds by 1 the number of valleys, deterministically). The last term in (5.40) can be estimated by observing that whenever $\eta \neq \vee$ then the number of sites $x$ such that $\eta_{x-1}=\eta_{x+1}=0$ is at most equal to the number of sites $x$ such that $\eta_{x-1}=\eta_{x+1}=1$. It follows that

$$
\sum_{x=1}^{L-1}\left[1_{\left\{\eta_{x-1}=\eta_{x+1}=0\right\}}-1_{\left\{\eta_{x-1}=\eta_{x+1}=1\right\}}\right] \leqslant 1_{\{\eta=\vee\}} .
$$

In particular,

$$
\mathcal{L} A(\eta) \leqslant-1_{\{\eta \neq \vee\}}
$$

This ends the proof of (5.40).

Finally, we prove the proposition for the system without the wall. Here the equilibrium measure $\mu$ is the uniform probability on all $2^{\frac{L}{2}}$ configurations

$$
\Omega_{0}=\left\{\eta \in \Omega_{L}: \quad \eta_{x}=0 \text { for all even } x\right\} .
$$

Let $\tau$ denote the hitting time of $\Omega_{0}$ for our process $\eta$ started in $\wedge$. Since $\lambda=\infty$ the process cannot exit $\Omega_{0}$ once it has entered. It is then obvious that $\tau$ coincides with the first time when the configuration started from $\wedge$ and evolving with the wall and $\lambda=\infty$ equals $\vee$, the (zigzag) minimal configuration satisfying the hard-wall constraint. Therefore, we already know from 
(5.41) that $\mathbb{E} \tau \leqslant L^{2} / 4$. Let - and + denote the minimal and maximal configurations in $\Omega_{0}$ respectively, and write $\eta^{-}(t), \eta^{+}(t)$ for the associated evolutions. The standard coupon-collector estimate gives that the coupling time $\tau^{\prime}$ of $\eta^{-}(t), \eta^{+}(t)$ satisfies $\mathbb{E} \tau^{\prime}=O(\log L)$ (there are $L / 2$ independent coordinates to be updated). Let now $\widetilde{\tau}$ denote the coupling time for $\eta^{\wedge}(t)$ and $\eta^{-}(t)$. We see that

$$
\begin{aligned}
\max _{\eta \in \Omega_{L}}\left\|P_{t}(\eta, \cdot)-\mu\right\|_{\text {var }} & \leqslant\left\|P_{t}(\wedge, \cdot)-P_{t}(-, \cdot)\right\|_{\text {var }} \\
& \leqslant \mathbb{P}(\widetilde{\tau} \geqslant t) \leqslant \frac{1}{t} \mathbb{E}[\widetilde{\tau}] \\
& \leqslant \frac{1}{t}\left(\mathbb{E}[\tau]+\mathbb{E}\left[\tau^{\prime}\right]\right) \leqslant \frac{1}{t}\left(L^{2} / 4+O(\log L)\right) .
\end{aligned}
$$

As before, this gives the mixing time bound $T_{\text {mix }} \leqslant L^{2}$, provided $L$ is sufficiently large.

\section{Proof of Theorem 3.5}

The statement concerning $\lambda \geqslant 1$ has been proven in Section 5.1 (spectral gap upper bound) and in Section 5.3 (mixing time lower bound), so we only need to prove (3.9). Before we do that, we give a heuristic argument which suggests that the $L^{-5 / 2}$ behavior in Theorem 3.5 might be the correct one.

\subsection{A heuristic justification of the $L^{-5 / 2}$ result}

Consider the model without wall and $\lambda<1$, and start the dynamics from a non-negative initial configuration $\xi$, e.g., $\xi=\wedge$. We know that the equilibrium measure $\mu_{L}^{\lambda}$ is symmetric under $\eta \leftrightarrow-\eta$ and, from (2.17), that $\mu_{L}^{\lambda}(\eta \leqslant 0)>0$ uniformly in $L$. Also, from the analysis of the model with the wall, we know that the dynamics restricted to configurations $\eta \geqslant 0$ (or to $\eta \leqslant 0$ ) relaxes in a time of order at most $O\left(L^{2} \log L\right)$. Therefore, it is reasonable that the relaxation time of our system without wall is of the same order as the first time $\tau$ such that $\eta_{x}^{\xi}(\tau) \leqslant 0$ for every $x$, provided that $\tau \gg L^{2} \log L$. On the other hand, it is plausible that the most convenient mechanism for the system to go from an initial configuration $\xi \geqslant 0$ to some $\eta \leqslant 0$ is the following:

1. first of all a "negative bubble" is formed close to one of the borders of the system (say, the left border). By "negative bubble at the left border" we mean that there exists $0<x \leqslant L / 2$ such that $\eta_{y} \leqslant 0$ for $y \leqslant 2 x$ and $\eta_{y} \geqslant 0$ for $y \geqslant 2 x$. The point $2 x$ will be referred to as the right-hand boundary of the bubble. Of course, when the bubble is first created one has $2 x=2$

2. the bubble grows until it occupies the whole $\Lambda$, i.e. until $2 x=L$.

Processes involving several bubbles or the formation of a bubble far away from the system boundaries would require that the configuration $\eta^{\xi}$ develops more zeros, and therefore they look much less likely in view of Eq. (2.15) and (2.19); at any rate, we neglect them. Now we introduce a simplified model which mimics the process of bubble formation and growth described above. We will implicitly assume that at any time $t$ the system is at equilibrium conditionally on the 


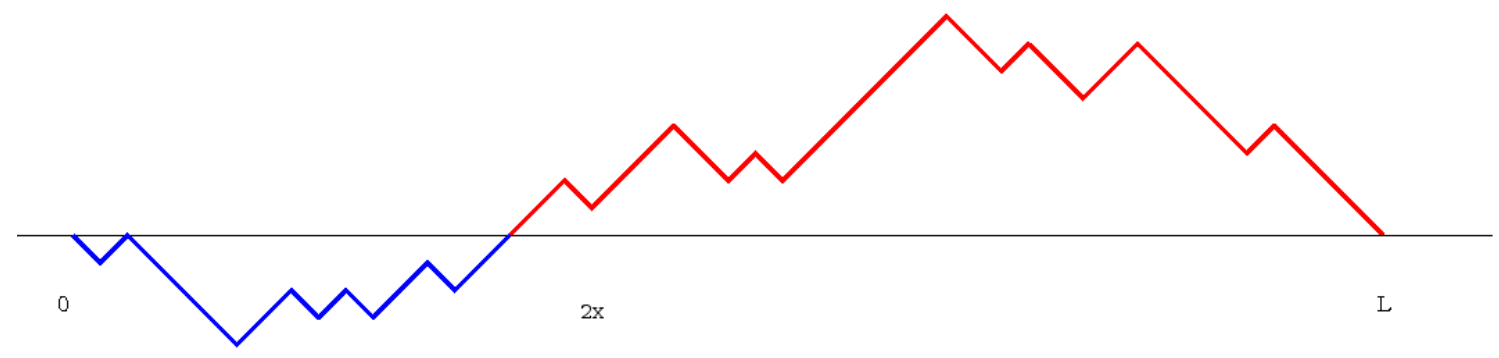

Figure 4: A typical configuration with a negative bubble at the left border. Apart from the point $2 x$ (the right-hand border of the bubble) the polymer has very few zeros, since the line is repulsive.

position, $2 x$, of the right-hand border of the bubble. Again, this is reasonable provided that $\tau \gg L^{2} \log L$.

Consider a birth-death process on $\{0, \ldots, L\}$ with invariant measure

$$
\nu(x):=Z^{-1} \frac{1}{(x \vee 1)^{3 / 2}((L-x) \vee 1)^{3 / 2}},
$$

where $Z=Z(L)$ normalizes $\nu(\cdot)$ to 1 . It is clear that $Z \approx L^{-3 / 2}$ and

$$
\nu(x)=\nu(L-x) \approx(x \vee 1)^{-3 / 2} \text { if } x \leqslant L / 2,
$$

where $A \approx B$ means that there exists a universal constant $c$ such that $(1 / c) \leqslant A / B \leqslant c$. We consider a Metropolis dynamics where the "birth" rate, $b(x)$, of jump from $x$ to $x+1$ is given for $x<L$ by $\min (1, \nu(x+1) / \nu(x))$, while the death rates are uniquely determined by the requirement that $\nu(\cdot)$ be reversible.

The connection of this dynamics with the "bubble dynamics" discussed above is obvious if one interprets $2 x$ as the rightmost point of a bubble in a system of length $2 L$, in view of

$$
\mu_{2 L}^{\lambda}\left(\eta_{y} \leqslant 0 \text { for } y<2 x ; \eta_{y} \geqslant 0 \text { for } y>2 x\right)=\frac{Z_{2 x}^{+}(\lambda) Z_{2(L-x)}^{+}(\lambda)}{Z_{2 L}(\lambda)}
$$

and of Theorem 2.1.

The following two observations will be useful in a while:

$$
\sum_{y=x}^{L} \nu(y) \approx 1 \approx \sum_{y=0}^{x} \nu(y)
$$

and

$$
b(x) \approx 1 .
$$

We will estimate how the inverse spectral gap of the birth-death process, gap $(L)^{-1}$, grows with $L$ applying a method of Hardy inequalities due to L. Miclo [19]. For this we need some additional 
notation, and we define for $0 \leqslant i \leqslant L$

$$
\begin{aligned}
B_{+}(i) & :=\sup _{x>i}\left(\sum_{y=i+1}^{x} \frac{1}{\nu(y) b(y)}\right) \sum_{y \geqslant x} \nu(y) \\
B_{-}(i) & :=\sup _{x<i}\left(\sum_{y=x}^{i-1} \frac{1}{\nu(y) b(y)}\right) \sum_{y \leqslant x} \nu(y) \\
B & :=\min _{0 \leqslant i \leqslant L}\left(B_{+}(i) \vee B_{-}(i)\right),
\end{aligned}
$$

with the convention that $B_{+}(L)=B_{-}(0)=0$. Then, Proposition 3.1 of [19] says that

$$
\frac{B}{2} \leqslant \operatorname{gap}(L)^{-1} \leqslant 4 B
$$

In view of (6.4) and (6.5), if we are only interested in the order of magnitude of the inverse spectral gap as a function of $L$ and not in precise constants, we can replace $b(y), \sum_{y} \geqslant x$ and $\sum_{y \leqslant x} \nu(y)$ by 1 in (6.6) and (6.7). Using (6.2), one finds

$$
B_{+}(i) \approx B_{-}(L-i) \approx \begin{cases}L^{5 / 2} & \text { if } i \leqslant L / 2 \\ (L-i+1)^{5 / 2} & \text { if } i \geqslant L / 2\end{cases}
$$

which immediately implies that $\operatorname{gap}(L)^{-1} \approx B \approx L^{5 / 2}$. Note that, in contrast with Theorem 3.5, no spurious logarithmic factor appears. Note also that the equilibration time for this birthdeath process is indeed much larger than $L^{2} \log L$, as required for the heuristic argument to be consistent, see discussion before Eq. (6.1).

\subsection{Proof of bound $(3.9)$}

We need some preliminary notation. Let $w: \mathbb{R} \ni x \mapsto w(x) \in[0,1]$ be a smooth function such that $w(x)=1$ for $x \leqslant-1$ and $w(x)=0$ for $x>1$. Recall the definition (5.39) of $A(\eta)$.

Theorem 6.1. Let $\lambda<1$ and define

$$
f(\eta):=w\left(\frac{A(\eta)}{L^{3 / 2}(\log L)^{-3}}\right) .
$$

There exists $c(\lambda, w)<\infty$ such that

$$
\frac{\mathcal{E}(f, f)}{\operatorname{Var}(f)}<c(\lambda, w) \frac{(\log L)^{8}}{L^{5 / 2}}
$$

As a consequence, we will deduce that if we start from the maximal configuration $\wedge$ then at any given time $t \ll L^{5 / 2} /(\log L)^{8}$ the area $A\left(\eta^{\wedge}(t)\right)$ is larger than $L^{3 / 2} /(\log L)^{3}$ with large probability. More precisely:

Proposition 6.2. For every $\lambda<1$

$$
\liminf _{\substack{L \rightarrow \infty, t=o\left(L^{5 / 2}(\log L)^{-8}\right)}} \mathbb{P}\left(A\left(\eta^{\wedge}(t)\right) \geqslant L^{3 / 2}(\log L)^{-3}\right)=1 .
$$


Of course, since at equilibrium $\mu_{L}^{\lambda}(A \leqslant 0) \geqslant 1 / 2$, this implies directly that the mixing time in this situation is at least $\Omega\left(L^{5 / 2}(\log L)^{-8}\right)$.

Proof of Theorem 6.1 For notational simplicity let $\varepsilon_{L}:=(\log L)^{-3}$. We put also

$$
b_{L}:=(\log L)^{-8 / 3}
$$

and

$$
\gamma_{L}:=(\log L)^{-7 / 6}
$$

It is easy to show that the variance of $f$ converges to $1 / 4$ for $L \rightarrow \infty$. Indeed, for $L$ large the function $w\left(A(\eta) /\left(L^{3 / 2} \varepsilon_{L}\right)\right)$ takes the value 1 with $\mu_{L}^{\lambda}$-probability $1 / 2+o(1)$ and the value 0 also with probability $1 / 2+o(1)$. This is quite intuitive from the properties of the delocalized phase discussed in section 2.1.2, but more precisely it follows from

$$
\begin{aligned}
1 / 2 \geqslant & \mu_{L}^{\lambda}\left(A(\eta) \geqslant L^{3 / 2} \varepsilon_{L}\right)=\mu_{L}^{\lambda}\left(A(\eta) \leqslant-L^{3 / 2} \varepsilon_{L}\right) \\
\geqslant & \frac{1}{2} \mu_{L}^{\lambda}\left(\nexists x: L b_{L}<x<L-L b_{L}, \eta_{x}=0\right) \\
& \times \mu_{L}^{\lambda}\left(|A(\eta)| \geqslant L^{3 / 2} \varepsilon_{L} \mid \nexists x: L b_{L}<x<L-L b_{L}, \eta_{x}=0\right)
\end{aligned}
$$

together with Eq. (2.19) and the fact that the last factor in the right-hand side is bounded below by $(1-2 / L)$ for large $L$, (cf. Lemma 6.3 below and the subsequent discussion). Therefore,

$$
\operatorname{Var}(f)=1 / 4+o(1)
$$

As for the Dirichlet form,

$$
\mathcal{E}(f, f) \leqslant 4 \frac{\left(\max _{x \in[-1,1]}\left|w^{\prime}(x)\right|\right)^{2}}{L^{2} \varepsilon_{L}^{2}} \mu_{L}^{\lambda}\left(|A(\eta)| \leqslant L^{3 / 2} \varepsilon_{L}\right) .
$$

A factor $L$ comes from the sum over $x$ in $(2.29)$, while the factor $L^{-3} / \varepsilon_{L}^{2}$ originates from

$$
\left|f(\eta)-Q_{x} f(\eta)\right| \leq \frac{2}{\varepsilon_{L} L^{3 / 2}} \times \max _{x \in\left[\frac{A(\eta)-1}{L^{3 / 2} \varepsilon_{L}}, \frac{A(\eta)+1}{L^{3 / 2} \varepsilon_{L}}\right]}\left|w^{\prime}(x)\right| .
$$

In order to conclude the proof, it is therefore sufficient to prove that

$$
\mu_{L}^{\lambda}\left(|A(\eta)| \leqslant L^{3 / 2} \varepsilon_{L}\right)=O\left(L^{-1 / 2}(\log L)^{2}\right)
$$

To this end, observe first of all that

$$
\begin{aligned}
\mu_{L}^{\lambda}\left(|A(\eta)| \leqslant L^{3 / 2} \varepsilon_{L}\right) & \leqslant \mu_{L}^{\lambda}\left(\exists x: L b_{L}<x<L-L b_{L}, \eta_{x}=0\right) \\
& +\mu_{L}^{\lambda}\left(|A(\eta)| \leqslant L^{3 / 2} \varepsilon_{L} ; \nexists x: L b_{L}<x<L-L b_{L}, \eta_{x}=0\right)
\end{aligned}
$$

The first term in the right-hand side of $(6.18)$ is of order $O\left(L^{-1 / 2}(\log L)^{4 / 3}\right)$, thanks to (2.19) and to the definition of $b_{L}$. As for the second one, decompose for convenience

$$
\begin{aligned}
A(\eta) & =\sum_{1 \leqslant x<L b_{L}} \eta_{x}+\sum_{L b_{L} \leqslant x \leqslant L-L b_{L}} \eta_{x}+\sum_{L b_{L}<x<L} \eta_{x} \\
& =: A^{(1)}(\eta)+A^{(2)}(\eta)+A^{(3)}(\eta) .
\end{aligned}
$$

The key estimate we need is 
Lemma 6.3. For L sufficiently large one has

$$
\mu_{L}^{\lambda}\left(\left|A^{(1)}(\eta)\right|+\left|A^{(3)}(\eta)\right| \geqslant(1 / 2) L^{3 / 2} \varepsilon_{L}\right) \leqslant \frac{1}{L}
$$

and

$$
\mu_{L}^{\lambda}\left(\left|A^{(2)}(\eta)\right| \leqslant 2 L^{3 / 2} \varepsilon_{L} ; \nexists x: L b_{L}<x<L-L b_{L}, \eta_{x}=0\right) \leqslant \frac{1}{L} .
$$

Indeed, thanks to the lemma we obtain immediately from (6.18)

$$
\mu_{L}^{\lambda}\left(|A(\eta)| \leqslant L^{3 / 2} \varepsilon_{L}\right) \leqslant O\left(L^{-1 / 2}(\log L)^{2}\right)+2 / L
$$

which concludes the proof of the theorem.

Proof of Lemma 6.3. The proof of (6.19) is easy. We start by observing that

$$
\begin{aligned}
\mu_{L}^{\lambda}\left(\left|A^{(1)}(\eta)\right| \geqslant(1 / 4) L^{3 / 2} \varepsilon_{L}\right) & \leqslant \mu_{L}^{\lambda}\left(\max _{x<L b_{L}}\left|\eta_{x}\right| \geqslant(1 / 4) L^{1 / 2} \frac{\varepsilon_{L}}{b_{L}}\right) \\
& =\mu_{L}^{+, 2 \lambda}\left(\max _{x<L b_{L}} \eta_{x} \geqslant(1 / 4) L^{1 / 2} \frac{\varepsilon_{L}}{b_{L}}\right) \\
& \leqslant \mu_{L}^{+, 0}\left(\max _{x<L b_{L}} \eta_{x} \geqslant(1 / 4) L^{1 / 2} \frac{\varepsilon_{L}}{b_{L}}\right)
\end{aligned}
$$

where we used monotonicity (say, FKG) in the last inequality. Since $\mu_{L}^{+, 0}(\cdot)=\mathbf{P}\left(\cdot \mid \eta_{L}=0, \eta_{x}>\right.$ $0 \forall 1<x<L)$ where we recall that $\mathbf{P}(\cdot)$ the law of the one-dimensional simple random walk started at 0 , we have

$$
\mu_{L}^{\lambda}\left(\left|A^{(1)}(\eta)\right| \geqslant(1 / 4) L^{3 / 2} \varepsilon_{L}\right) \leqslant \frac{\mathbf{P}\left(\max _{x<L b_{L}} \eta_{x} \geqslant(1 / 4) L^{1 / 2} \varepsilon_{L} / b_{L}\right)}{\mathbf{P}\left(\eta_{L}=0 ; \eta_{x}>0 \forall 1<x<L\right)} .
$$

Now, for the denominator we employ (2.18), while for the numerator we observe that

$$
\begin{array}{r}
\mathbf{P}\left(\max _{x<L b_{L}} \eta_{x} \geqslant(1 / 4) L^{1 / 2} \varepsilon_{L} / b_{L}\right)=\sum_{\ell \geqslant(1 / 4) L^{1 / 2} \varepsilon_{L} / b_{L}} \mathbf{P}\left(\max _{x<L b_{L}} \eta_{x}=\ell\right) \\
=\sum_{\ell \geqslant(1 / 4) L^{1 / 2} \varepsilon_{L} / b_{L}} \max \left(\mathbf{P}\left(\eta_{L} b_{L}=\ell\right), \mathbf{P}\left(\eta_{L} b_{L}=\ell+1\right)\right)
\end{array}
$$

where we used [8, Sec. III.7,Th. 1] in the last equality. From this one sees that

$$
\mu_{L}^{\lambda}\left(\left|A^{(1)}(\eta)\right| \geqslant(1 / 4) L^{3 / 2} \varepsilon_{L}\right) \leqslant \frac{1}{2 L}
$$

for $L$ large. Since $A^{(1)}(\eta)$ and $A^{(3)}(\eta)$ are equally distributed, this proves (6.19).

As for (6.20), we note (using also the symmetry $\eta \leftrightarrow-\eta$ ) that the left-hand side is bounded above by

$$
\mu_{L}^{\lambda}\left(0<A^{(2)}(\eta) \leqslant 2 L^{3 / 2} \varepsilon_{L} \mid \eta_{x}>0 \forall L b_{L}<x<L-L b_{L}\right)
$$


which by FKG is itself bounded above for $L$ large by

$$
\mathbf{P}\left(A_{\ell}(\eta) \leqslant 4 \ell^{3 / 2} \varepsilon_{\ell} \mid \eta_{\ell}=0 ; \eta_{x}>0 \forall 1<x<\ell\right)
$$

where we put $\ell:=\ell(L):=L-2\left\lfloor L b_{L}\right\rfloor$ and for clarity of notation $A_{\ell}(\eta):=\sum_{x \leqslant \ell} \eta_{x}$. Letting $M_{\ell}:=\left\lceil\ell \gamma_{\ell}\right\rceil$, one can bound above $(6.25)$ by

$$
\begin{aligned}
& \mathbf{P}\left(\max _{x \leqslant \ell} \eta_{x} \leqslant \sqrt{M_{\ell}} \mid \eta_{\ell}=0 ; \eta_{x}>0 \forall 1<x<\ell\right) \\
& +\mathbf{P}\left(\max _{x \leqslant \ell} \eta_{x}>\sqrt{M_{\ell}} ; A_{\ell}(\eta) \leqslant 4 \ell^{3 / 2} \varepsilon_{\ell} \mid \eta_{\ell}=0 ; \eta_{x}>0 \forall 1<x<\ell\right) .
\end{aligned}
$$

Using monotonicity twice, the first term of (6.26) is easily bounded above by

$$
\begin{aligned}
& \mathbf{P}\left(\max _{x \leqslant \ell} \eta_{x} \leqslant \sqrt{M_{\ell}} \mid \eta_{1}>0, \ldots, \eta_{\ell-1}>0 ; \eta_{\ell}=0 ; \eta_{2 j} M_{\ell}=2, j \leqslant\left\lfloor 1 /\left(4 \gamma_{\ell}\right)\right\rfloor\right) \\
& \leqslant\left[\mathbf{P}\left(\eta_{M_{\ell}} \leqslant \sqrt{M_{\ell}} \mid \eta_{1}>0, \ldots, \eta_{2 M_{\ell}-1}>0 ; \eta_{2 M_{\ell}}=0\right)\right]^{1 /\left(4 \gamma_{\ell}\right)} \\
& \leqslant\left[\mathbf{P}\left(\eta_{M_{\ell}} \leqslant \sqrt{M_{\ell}} \mid \eta_{2 M_{\ell}}=0\right)\right]^{1 /\left(4 \gamma_{\ell}\right)}
\end{aligned}
$$

Since $n^{-1 / 2} \eta_{n / 2}$ converges weakly for $n \rightarrow \infty$ under $\mathbf{P}\left(\cdot \mid \eta_{n=0}\right)$ to a non-degenerate Gaussian random variable (the Brownian Bridge at time 1/2), the probability in the last expression is strictly smaller than 1 uniformly in $\ell$, and therefore the first term in (6.26) is smaller than $1 / L$ for $L$ large.

As for the second term in (6.26), we note that the conditions on $\max _{x} \leqslant \ell \eta_{x}$ and on $A_{\ell}(\eta)$ imply that there exist $1<x, y<\ell$ such that $|x-y| \leqslant 4 \ell \varepsilon_{\ell} / \sqrt{\gamma_{\ell}}$ and $\left|\eta_{x}-\eta_{y}\right| \geqslant(1 / 2) \sqrt{M_{\ell}}$ (just take as $x$ the position of the maximum of $\eta$ ). As a consequence, using (2.18) one can bound above the second term in $(6.26)$ by

$$
c \ell^{3 / 2} \mathbf{P}\left(\exists 1<x<y<\ell:|x-y| \leqslant 4 \ell \varepsilon_{\ell} / \sqrt{\gamma_{\ell}},\left|\eta_{x}-\eta_{y}\right| \geqslant(1 / 2) \ell^{1 / 2} \sqrt{\gamma_{\ell}}\right)
$$

and for this quantity the upper bound $1 / \ell$ for $\ell$ large follows immediately from standard simplerandom-walk estimates. The factor $\ell^{3 / 2}$ arises from the estimate (2.18).

Proof of Proposition 6.2. Let $w_{+}: \mathbb{R} \ni x \mapsto w_{+}(x) \in[0,1]$ be a smooth function such that $w_{+}(x)=0$ for $x \leqslant 1 / 2$ and $w_{+}(x)=1$ for $x \geqslant 1$, and define $w_{-}($.$) via w_{-}(x)=w_{+}(-x)$. We put $f_{ \pm}(\eta):=w_{ \pm}\left(A(\eta) / L^{3 / 2} \varepsilon_{L}\right)$ where, as in the proof of Theorem 6.1, $\varepsilon_{L}:=(\log L)^{-3}$. The proof of Theorem 6.1 can be repeated essentially without changes to show that

$$
\mathcal{E}\left(f_{ \pm}, f_{ \pm}\right)=O\left(L^{-5 / 2}(\log L)^{8}\right)
$$

To begin the proof of (6.13), observe that by monotonicity

$$
\mathbb{P}\left(A\left(\eta^{\wedge}(t)\right) \geqslant L^{3 / 2} \varepsilon_{L}\right) \geqslant \frac{\int d \mu_{L}^{\lambda}(\xi)\left(f_{+}(\xi)\right)^{2} \mathbb{P}\left(A\left(\eta^{\xi}(t)\right) \geqslant L^{3 / 2} \varepsilon_{L}\right)}{\mu_{L}^{\lambda}\left(\left(f_{+}\right)^{2}\right)} .
$$


It is immediate to realize that

$$
\mu_{L}^{\lambda}\left(\left(f_{+}\right)^{2}\right)=\mu_{L}^{\lambda}\left(f_{+}\right)+o(1)=1 / 2+o(1)
$$

for $L \rightarrow \infty$. Using reversibility of dynamics and Cauchy-Schwarz in the numerator one obtains then

$$
\begin{aligned}
\mathbb{P}\left(A\left(\eta^{\wedge}(t)\right) \geqslant L^{3 / 2} \varepsilon_{L}\right) \geqslant & (2+o(1)) \int d \mu_{L}^{\lambda}(\xi) \mathbf{1}_{\left\{A(\xi) \geqslant L^{3 / 2} \varepsilon_{L}\right\}}\left[\left(P_{t} f_{+}\right)(\xi)\right]^{2} \\
= & (2+o(1)) \mu_{L}^{\lambda}\left[\left(P_{t} f_{+}\right)^{2}\right] \\
- & (2+o(1)) \int d \mu_{L}^{\lambda}(\xi) \mathbf{1}_{\left\{A(\xi)<L^{3 / 2} \varepsilon_{L}\right\}}\left[\left(P_{t} f_{+}\right)(\xi)\right]^{2}
\end{aligned}
$$

We will show later that

$$
\operatorname{Var}\left(P_{t} f_{+}\right) \geqslant \operatorname{Var}\left(f_{+}\right) e^{-2 t \frac{c(\lambda)(\log L)^{8}}{L^{5 / 2}}}
$$

From (6.30) one then deduces that

$$
(2+o(1)) \mu_{L}^{\lambda}\left[\left(P_{t} f_{+}\right)^{2}\right] \geqslant 1+o(1)
$$

for $t=o\left(L^{5 / 2} /(\log L)^{8}\right)$. As for the integral in (6.31), rewrite it as

$$
\int d \mu_{L}^{\lambda}(\xi) \mathbf{1}_{\left\{|A(\xi)|<L^{3 / 2} \varepsilon_{L}\right\}}\left[\left(P_{t} f_{+}\right)(\xi)\right]^{2}+\int d \mu_{L}^{\lambda}(\xi) \mathbf{1}_{\left\{A(\xi) \leqslant-L^{3 / 2} \varepsilon_{L}\right\}}\left[\left(P_{t} f_{+}\right)(\xi)\right]^{2} .
$$

The first term is $o(1)$ as follows from (6.17) plus the fact that $f_{+}$is bounded. The second one, on the other hand, is bounded above by

$$
\mu_{L}^{\lambda}\left(f_{-} P_{t} f_{+}\right)
$$

(indeed, recall that $\left\|f_{+}\right\| \leqslant 1$.) It is obvious from the definition of $f_{ \pm}$that this integral vanishes at $t=0$. To show that (6.35) is $o(1)$ we evaluate the $t$-derivative of it: using reversibility and Cauchy-Schwarz,

$$
\begin{aligned}
\left|\frac{d}{d t} \mu_{L}^{\lambda}\left(f_{-} P_{t} f_{+}\right)\right| & =\left|\mu_{L}^{\lambda}\left(f_{-}(-\mathcal{L}) P_{t} f_{+}\right)\right| \\
& =\left|\mu_{L}^{\lambda}\left((-\mathcal{L})^{1 / 2} f_{-}(-\mathcal{L})^{1 / 2} P_{t} f_{+}\right)\right| \\
& \leqslant \sqrt{\mathcal{E}\left(f_{-}, f_{-}\right) \mathcal{E}\left(f_{+}, f_{+}\right)}=O\left(L^{-5 / 2}(\log L)^{8}\right) .
\end{aligned}
$$

We can therefore conclude that (6.34) is $o(1)$ for $t=o\left(L^{5 / 2} /(\log L)^{8}\right)$.

Finally, we prove (6.32). This is a simple consequence of the general inequality

$$
\operatorname{Var}\left(P_{t} f\right) \geqslant \operatorname{Var}(f) e^{-2 t \frac{\mathcal{E}(f, f)}{\operatorname{Var}(f)}}
$$

which holds for every $f$ thanks to the spectral theorem plus Jensen's inequality, and of Eqs. (6.28) and (6.30). 


\section{$7 \quad$ Further results in the localized phase}

In this section we prove Theorem 3.3 and Theorem 3.6. All our arguments below refer to the system with the wall with $\lambda>2$.

\subsection{Proof of Theorem 3.3}

Recall the definition (2.3) of the equilibrium measure $\mu_{L}^{+, \lambda}$ and set

$$
U(L, t):=\max _{0<x<L}\left(\mu_{L}^{+, \lambda}\left(\eta_{x}\right)-\mathbb{E}_{L}\left(\eta_{x}^{\vee}(t)\right) \geqslant 0,\right.
$$

where for later convenience we indicated explicitly the $L$-dependence in the average over the process. Non-negativity follows from monotonicity. Also, from monotonicity and Markov's inequality we have

$$
\left\|P_{t}(\vee, \cdot)-\mu_{L}^{+, \lambda}\right\|_{\text {var }} \leqslant \frac{1}{2} L U(L, t) .
$$

Let $\ell=\ell(L):=2\left\lfloor c_{0} \log L\right\rfloor \in 2 \mathbb{N}$ where $c_{0}$ will be chosen sufficiently large later. Thanks to the exponential decay of correlations (cf. Lemma 2.2 and subsequent discussion), one has for every $\ell / 2 \leqslant x \leqslant L-\ell / 2$

$$
0 \leqslant \mu_{L}^{+, \lambda}\left(\eta_{x}\right)-\mu_{\ell}^{+, \lambda}\left(\eta_{\ell / 2}\right) \leqslant c e^{-\ell / c}
$$

Here and below we write $c$ for a suitable constant, whose value may vary from line to line. For $1 \leqslant x \leqslant \ell / 2$ one has instead

$$
0 \leqslant \mu_{L}^{+, \lambda}\left(\eta_{x}\right)-\mu_{\ell}^{+, \lambda}\left(\eta_{x}\right) \leqslant c e^{-\ell / c},
$$

and for $L-\ell / 2 \leqslant x \leqslant L$

$$
0 \leqslant \mu_{L}^{+, \lambda}\left(\eta_{x}\right)-\mu_{\ell}^{+, \lambda}\left(\eta_{x-L+\ell}\right) \leqslant c e^{-\ell / c} .
$$

If e.g. $\ell / 2 \leqslant x \leqslant L-\ell / 2$, then (7.3) implies

$$
\begin{aligned}
0 & \leqslant \mu_{L}^{+, \lambda}\left(\eta_{x}\right)-\mathbb{E}_{L}\left(\eta_{x}^{\vee}(t)\right) \leqslant c e^{-\ell / c}+\mu_{\ell}^{+, \lambda}\left(\eta_{\ell / 2}\right)-\mathbb{E}_{L}\left(\eta_{x}^{\vee}(t)\right) \\
& \leqslant c e^{-\ell / c}+\mu_{\ell}^{+, \lambda}\left(\eta_{\ell / 2}\right)-\mathbb{E}_{\ell}\left(\eta_{\ell / 2}^{\vee}(t)\right),
\end{aligned}
$$

where we used again monotonicity in the last inequality. For $x \notin[\ell / 2, L-\ell / 2]$ one obtains analogous bounds from Eqs. (7.4) -(7.5). As a consequence, one concludes that for every $t \geqslant 0$

$$
U(L, t) \leqslant c e^{-\ell(L) / c}+U(\ell(L), t) .
$$

From (4.1) it follows that for every $n \in 2 \mathbb{N}$

$$
\left|\mu_{n}^{+, \lambda}\left(\eta_{x}\right)-\mathbb{E}_{n}\left(\eta_{x}^{\xi}(t)\right)\right| \leqslant c n^{3} e^{-t /\left(c n^{2}\right)},
$$

for every $x \leqslant n, t \geqslant 0$ and every initial condition $\xi$. Therefore, (7.7) implies

$$
U(L, t) \leqslant \frac{c}{L^{c_{0} / c}}+c c_{0}^{3}(\log L)^{3} e^{-t /\left(c c_{0}^{2}(\log L)^{2}\right)} .
$$


If $t \geqslant t_{0}(L):=c_{1}(\log L)^{3}$ with $c_{1}$ sufficiently large, then

$$
U(L, t) \leqslant \frac{c}{L^{c_{0} / c}}
$$

Finally, if we choose $c_{0}$ sufficiently large in the definition of $\ell(L)$, it follows from (7.8) and (7.2) that for $t \geqslant t_{0}(L)$ the variation distance between $\mu_{L}^{+, \lambda}($.$) and the distribution of \eta^{\vee}(t)$ is $o(1)$, and Eq. (3.7) is proven.

Let us now turn to the proof of the lower bound on the equilibration time starting from $\vee$. It is possible to apply the ideas of [15] to prove that the dynamics starting from $\vee$ takes at least a time of order $\log L$ to relax to equilibrium, but we shall prove the stronger statement (3.8). To begin, we define $\mathcal{C}$ to be the set $\mathcal{C}:=\{2 j\lfloor\sqrt{L}\rfloor, j \leqslant\lfloor\sqrt{L}\rfloor / 2-1\}$ and

$$
f(\eta):=\frac{1}{|\mathcal{C}|} \sum_{x \in \mathcal{C}} 1_{\eta_{x}=0} .
$$

Using the exponential decay of correlations (Lemma 2.2) we see that

$$
\operatorname{Var}(f) \leqslant \frac{c}{\sqrt{L}}
$$

where the variance is computed w.r.t. $\mu_{L}^{+, \lambda}$. Next, we need the following estimate, whose proof will be given later.

Lemma 7.1. There exist positive constants $c_{0}=c_{0}(\lambda)$ and $L_{0}$ such that for every $x \in \mathcal{C}$, $0<t<\sqrt{L}$ and $L>L_{0}$ one has

$$
\mathbb{P}\left(\eta_{x}^{\vee}(t)=0\right)-\mu_{L}^{+, \lambda}\left(\eta_{x}=0\right) \geqslant c_{0} e^{-\sqrt{t} / c_{0}}
$$

In order to prove that at time $(\log L)^{2} / c$ the total variation distance from equilibrium is still $1+o(1)$, we introduce the set

$$
K:=\left\{\eta \in \Omega_{L}^{+}: f(\eta) \geqslant \mu_{L}^{+, \lambda}(f)+\left(c_{0} / 2\right) e^{-\sqrt{t} / c_{0}}\right\}
$$

where $c_{0}$ is the same as in (7.10), and we show that $\mu_{L}^{+, \lambda}(K)=o(1)$ while

$$
\mathbb{P}\left(\eta^{\vee}(t) \in K\right)=1+o(1)
$$

The first fact follows from (7.9) and Chebyshev's inequality:

$$
\mu_{L}^{+, \lambda}(K) \leqslant \frac{c}{c_{0}^{2} \sqrt{L}} e^{2 \sqrt{t} / c_{0}}=o(1)
$$

as $L \rightarrow \infty$, if $t \leqslant(\log L)^{2} / c$. As for (7.11), it is convenient to introduce a modified process, call it $\tilde{\eta}^{\vee}(t)$, which is just the original process started from $\vee$ but conditioned on the event that, for every $t \geqslant 0, \eta_{x}^{\vee}(t) \equiv \vee_{x}$ for every $x$ such that $\min \{|x-j|: j \in \mathcal{C}\} \geqslant \sqrt{L} / 2$ (in other words, the points at distance at least $\sqrt{L} / 2$ from $\mathcal{C}$ are kept at their initial values for all times). Denote by $\pi_{t}(\cdot)$ (respectively $\tilde{\pi}_{t}(\cdot)$ ) the marginal distribution of $\left\{\eta_{x}\right\}_{x \in \mathcal{C}}$ under the law of $\eta^{\vee}(t)$ (resp. the law of $\left.\tilde{\eta}^{\vee}(t)\right)$. The proof of the next claim is postponed for a moment. 


\section{Claim 7.2.}

$$
\left\|\pi_{t}(\cdot)-\tilde{\pi}_{t}(\cdot)\right\|_{\mathrm{var}} \leqslant c e^{-(\log L) \sqrt{L} / c}
$$

if $t \leqslant(\log L)^{2} / c$.

The same is actually true as long as $t \leqslant L^{1 / 2-\varepsilon}$, but we will not need that. Assuming the validity of (7.13) we are able to finish the proof of the theorem.

Observe that

$$
\operatorname{Var}_{\tilde{\pi}_{t}}(f) \leqslant \frac{c}{\sqrt{L}}
$$

since, from the way the modified dynamics is constructed, $\tilde{\eta}_{x}^{\vee}(t)$ is independent of $\tilde{\eta}_{y}^{\vee}(t)$ for $x, y \in \mathcal{C}$ with $x \neq y$. From Eqs. (7.13) and (7.14) and the fact that $\|f\|_{\infty}=1$ one deduces that

$$
\operatorname{Var}_{\pi_{t}}(f) \leqslant \frac{c}{\sqrt{L}}
$$

Thanks to (7.15) and (7.10), equation (7.11) is seen to hold for all $t \leqslant(\log L)^{2} / c$ by an application of Chebyshev's inequality.

Proof of Claim 7.2. This is based on a standard disagreement percolation argument, see e.g. [15, Sec. 3.1]. Consider $n$ IID Poisson clocks of rate 1 and let $p(n, t)$ be the probability that there is an increasing sequence of times $0<t_{1}<\ldots<t_{n}<t$ such that the clock labeled $i$ rings at time $t_{i}$. An standard computation gives that

$$
p(n, t)<\left(\frac{e t}{n}\right)^{n}
$$

On the other hand, it is immediate to realize that

$$
\mathbb{P}\left(\exists x \in \mathcal{C}: \eta_{x}^{\vee}(t) \neq \tilde{\eta}_{x}^{\vee}(t)\right) \leqslant 2|\mathcal{C}| p(\lfloor\sqrt{L} / 2\rfloor, t),
$$

from which Eq. (7.13) easily follows.

Proof of Lemma 7.1. Define, for $\ell \in 2 \mathbb{N}$, the set

$$
B_{x, \ell}:=\left\{\xi: \xi_{x+j}=\ell-|j| \text { for every }|j| \leqslant \ell\right\} .
$$

In other words, configurations $\xi \in B_{x, \ell}$ take in $\{x-\ell, \ldots, x+\ell\}$ the maximal value allowed by the constraint $\xi_{x \pm \ell}=0$ (see Fig. 5).

Let $\ell(t) \in 2 \mathbb{N}$ satisfy

$$
c_{1} \sqrt{t}<\ell(t)<2 c_{1} \sqrt{t},
$$

for some sufficiently large constant $c_{1}$ to be chosen later. Since we are in the localized phase,

$$
\mu_{L}^{+, \lambda}\left(B_{x, \ell(t)}\right) \geqslant c e^{-\sqrt{t} / c},
$$




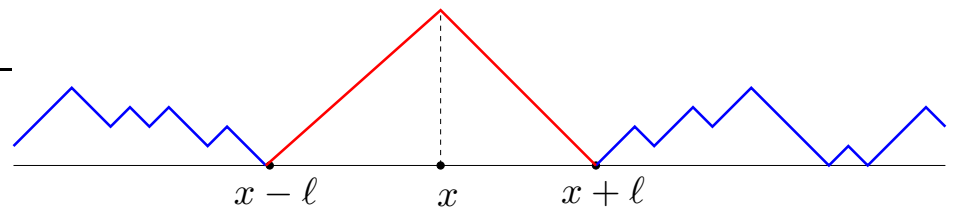

Figure 5: A typical path belonging to $B_{x, \ell}$.

uniformly in $L$. Indeed, it is not difficult to deduce from Lemma 2.2 that $\mu_{L}^{+, \lambda}\left(\eta_{a}=\eta_{b}=0\right)$ is bounded away from zero uniformly in $a, b, L \in 2 \mathbb{Z}$. On the other hand, from the definition of the model

$$
\mu_{L}^{+, \lambda}\left(B_{x, \ell(t)}\right)=\frac{\mu_{L}^{+, \lambda}\left(\eta_{x \pm \ell(t)=0}\right)}{Z_{2 \ell(t)}^{+}(\lambda)},
$$

from which the claim (7.20) follows through Theorem 2.1 .

Next, we write

$$
\mathbb{P}\left(\eta_{x}^{\vee}(t)=0\right)-\mu_{L}^{+, \lambda}\left(\eta_{x}=0\right)=\int d \mu_{L}^{+, \lambda}(\xi)\left[\mathbb{P}\left(\eta_{x}^{\vee}(t)=0\right)-\mathbb{P}\left(\eta_{x}^{\xi}(t)=0\right)\right] .
$$

Since the quantity which is being integrated in the right-hand side of (7.21) is non-negative by monotonicity, (7.20) implies

$$
\begin{aligned}
& \mathbb{P}\left(\eta_{x}^{\vee}(t)=0\right)-\mu_{L}^{+, \lambda}\left(\eta_{x}=0\right) \\
& \quad \geqslant c e^{-\sqrt{t} / c} \int d \mu_{L}^{+, \lambda}\left(\xi \mid \xi \in B_{x, \ell(t)}\right)\left[\mathbb{P}\left(\eta_{x}^{\vee}(t)=0\right)-\mathbb{P}\left(\eta_{x}^{\xi}(t)=0\right)\right] .
\end{aligned}
$$

Note that, if $\xi \in B_{x, \ell(t)}$ then $\eta^{\xi}(s)$ is stochastically higher, for every $s>0$, than the configuration $\hat{\eta}^{\xi}(s)$ which has law $\mathbb{P}\left(\cdot \mid \eta_{x \pm \ell(t)}^{\xi}(r)=0 \forall r \leqslant t\right)$. This holds in particular for $s=t$. Therefore,

$$
\mathbb{P}\left(\eta_{x}^{\vee}(t)=0\right)-\mu_{L}^{+, \lambda}\left(\eta_{x}=0\right) \geqslant c e^{-\sqrt{t} / c}\left[\mu_{L}^{+, \lambda}\left(\eta_{x}=0\right)-\mathbb{P}\left(\hat{\eta}_{x}^{\xi}(t)=0\right)\right]
$$

where it is clear that the last term is independent of the choice of $\xi \in B_{x, \ell(t)}$. Indeed, $\left\{\hat{\eta}_{x}^{\xi}(s)\right\}_{s} \geqslant 0$ depends only on the value of $\xi$ in the interval $\{x-\ell(t), x+\ell(t)\}$, on which however there is no choice once we require that $\xi \in B_{x, \ell(t)}$.

Next, we shall use the following estimate, the proof of which is postponed for a moment. Recall that $c_{1}$ is the constant defining $\ell(t)$ in (7.19).

Claim 7.3. For any $\varepsilon_{1}, \varepsilon_{2}>0$, there exists $C>0$ such that for all $c_{1} \geqslant C$ :

$$
\mathbb{P}\left(\hat{\eta}_{x}^{\xi}(t)<\left(1-\varepsilon_{1}\right) \ell(t)\right) \leqslant \varepsilon_{2}, \quad t \geqslant 0 .
$$


From (7.23), for any given $\varepsilon>0$, if $c_{1}$ is chosen sufficiently large as a function of $\varepsilon$, we have

$$
\mathbb{P}\left(\hat{\eta}_{x}^{\xi}(t)=0\right)<\varepsilon
$$

Choosing $\varepsilon<\mu_{L}^{+, \lambda}\left(\eta_{x}=0\right) / 2$, the desired estimate (7.10) follows. Note that this $\varepsilon>0$ can be chosen to be independent of $L$ since in the localized phase the probabilities $\mu_{L}^{+, \lambda}\left(\eta_{x}=0\right)$ are uniformly bounded away from zero. This ends the proof of the Lemma 7.1.

Proof of Claim 7.3. By monotonicity it is sufficient to prove the claim at $\lambda=\infty$. Let $\varphi_{L}(t)$ denote the height in the middle of the segment $\{0, \ldots, L\}$, at time $t$, of the usual process $\eta^{\wedge}(t)$. It suffices to prove that, for every $\varepsilon_{1}, \varepsilon_{2}>0$ there exists $\delta>0$ such that

$$
\mathbb{P}\left(\varphi_{L}(t) \leqslant\left(1-\varepsilon_{1}\right) \frac{L}{2}\right) \leqslant \varepsilon_{2}, \quad t \leqslant \delta L^{2} .
$$

This can be shown to follow from the argument in the proof of Theorem 5.5. Namely, let $B_{D}$ and $\xi(t)$ be the area and the auxiliary process defined there. Note that the geometric construction of Section 5.3 implies, in particular, that if $\varphi_{L}(t) \leqslant\left(1-\varepsilon_{1}\right) \frac{L}{2}$ then $B_{D}(\xi(t)) \leqslant\left(1-\varepsilon^{\prime}\right) B_{D}(\wedge)$ for some $\varepsilon^{\prime}>0$. Also, recall that $\mathbb{E} B_{D}(\xi(t)) \geqslant B_{D}(\wedge)-t$, so that

$$
\operatorname{Var}\left[B_{D}(\xi(t))\right]=\mathbb{E}\left[B_{D}(\xi(t))^{2}\right]-\mathbb{E}\left[B_{D}(\xi(t))\right]^{2} \leqslant B_{D}(\wedge)^{2}-\left[B_{D}(\wedge)-t\right]^{2} \leqslant 2 t B_{D}(\wedge) .
$$

Then the claim follows from an application of Chebyshev's inequality.

\subsection{Stretched exponential decay of local observables: Proof of Theorem 3.6}

We start with the proof of the upper bound (3.10). We first prove it under the extra assumption that $f$ is monotone decreasing and non-negative. For $\ell_{1}, \ell_{2} \in 2 \mathbb{Z}$ with $\ell_{1}<\ell_{2}$ let

$$
\mu_{\ell_{1}, \ell_{2}}^{+}(\cdot):=\mu_{\infty}^{+}\left(\cdot \mid \eta_{\ell_{1}}=\eta_{\ell_{2}}=0\right)
$$

denote the equilibrium measure with zero boundary conditions at $\ell_{1}$ and $\ell_{2}$. From Lemma 2.2 it follows that if we choose $\ell_{i}$ such that $\mathcal{S}_{f} \subset\left\{\ell_{1}, \ldots, \ell_{2}\right\}$, then

$$
\left|\mu_{\infty}^{+}(f)-\mu_{\ell_{1}, \ell_{2}}^{+}(f)\right| \leqslant c\|f\|_{\infty}\left[e^{-d\left(\mathcal{S}_{f},\left\{\ell_{1}\right\}\right) / c}+e^{-d\left(\mathcal{S}_{f},\left\{\ell_{2}\right\}\right) / c}\right] .
$$

By positivity and monotonicity of $f$ one has for every $t \geqslant 0$ that

$$
\mu_{\infty}^{+}\left(\left(P_{t} f\right)^{2}\right) \leqslant \mu_{\ell_{1}, \ell_{2}}^{+}\left(\left(P_{t} f\right)^{2}\right) \leqslant \int d \mu_{\ell_{1}, \ell_{2}}^{+}(\xi)\left(\mathbb{E}\left(f\left(\eta^{\xi}(t)\right) \mid \eta_{\ell_{1}}^{\xi}(r)=\eta_{\ell_{2}}^{\xi}(r)=0 \forall r \leqslant t\right)\right)^{2} .
$$

Therefore, using the lower bound on the spectral gap given by (3.1), one finds

$$
\begin{aligned}
\operatorname{Var}_{\mu_{\infty}^{+}}\left(P_{t} f\right) \leqslant & \operatorname{Var}_{\mu_{\ell_{1}, \ell_{2}}^{+}}(f) e^{-t /\left[c\left(\ell_{2}-\ell_{1}\right)^{2}\right]} \\
& +c\|f\|_{\infty}^{2}\left[e^{-d\left(\mathcal{S}_{f},\left\{\ell_{1}\right\}\right) / c}+e^{-d\left(\mathcal{S}_{f},\left\{\ell_{2}\right\}\right) / c}\right] .
\end{aligned}
$$

We may choose $\ell_{i}, i=1,2$ such that

$$
t^{1 / 3} \leqslant d\left(\mathcal{S}_{f},\left\{\ell_{i}\right\}\right)<2 t^{1 / 3}
$$


which gives $\left(\ell_{2}-\ell_{1}\right)<c t^{1 / 3}$ for $t^{1 / 3}>\operatorname{Diam}\left(\mathcal{S}_{f}\right)$. Since $\operatorname{Var}_{\mu_{\ell_{1}, \ell_{2}}^{+}}(f) \leqslant\|f\|_{\infty}^{2},(7.26)$ proves (3.10) for all $t$ such that $t>\left(\operatorname{Diam}\left(\mathcal{S}_{f}\right)\right)^{3}$. If $t$ is smaller than that then we obtain again the claimed bound by adjusting the constant $C_{f}$. This proves (3.10) for $f$ bounded, local, non-negative and decreasing.

To prove the claim for any bounded local $f$ we first introduce a cutoff parameter $\ell_{0}$ and rewrite $f$ as $f=f_{0}+f_{1}$ where $f_{0}(\eta)=f(\eta) 1_{E}$ and $f_{1}(\eta)=f(\eta) 1_{E^{c}}$ with $E$ representing the event $\left\{\max _{x \in \mathcal{S}_{f}} \eta_{x} \leqslant \ell_{0}\right\}$. Observe that

$$
\operatorname{Var}_{\mu_{\infty}^{+}}\left(P_{t} f\right) \leqslant 2 \operatorname{Var}_{\mu_{\infty}^{+}}\left(P_{t} f_{0}\right)+2\|f\|_{\infty}^{2} \mu_{\infty}^{+}\left(E^{c}\right) .
$$

Since in the localized phase the height at any point has an exponential tail, one has $\mu_{\infty}^{+}\left(E^{c}\right) \leqslant c\left|\mathcal{S}_{f}\right| e^{-\ell_{0} / c}$, where $\left|\mathcal{S}_{f}\right|$ stands for the cardinality of $\mathcal{S}_{f}$. Let now $\Omega_{0}$ denote the set of all possible values of the configuration $\left\{\eta_{x}, x \in \mathcal{S}_{f}\right\}$ that are compatible with the constraint $\eta \in E$. Note that its cardinality $\left|\Omega_{0}\right|$ is at most $C \ell_{0}$, for some constant $C$ depending on $\mathcal{S}_{f}$. We can write $1_{\left\{\eta_{x}=\sigma_{x}\right\}}=1_{\left\{\eta_{x} \leqslant \sigma_{x}\right\}}-1_{\left\{\eta_{x}<\sigma_{x}\right\}}$ and expand

$$
f_{0}(\eta)=\sum_{\sigma \in \Omega_{0}} f(\sigma) \prod_{x \in \mathcal{S}_{f}} 1_{\left\{\eta_{x}=\sigma_{x}\right\}}=\sum_{\sigma \in \Omega_{0}} \sum_{A \subset \mathcal{S}_{f}}(-1)^{\left|\mathcal{S}_{f} \backslash A\right|} f(\sigma) g_{\sigma, A},
$$

where $g_{\sigma, A}:=\prod_{x \in A, y \in \mathcal{S}_{f} \backslash A} 1_{\left\{\eta_{x} \leqslant \sigma_{x}\right\}} 1_{\left\{\eta_{y}<\sigma_{y}\right\}}$. The latter is a bounded local, non-negative and decreasing function to which the argument leading to (7.26) applies. Adjusting the constant $C_{f}$ we may therefore estimate

$$
\begin{aligned}
\operatorname{Var}_{\mu_{\infty}^{+}}\left(P_{t} f_{0}\right) & \leqslant C_{f} \ell_{0} \sum_{\sigma \in \Omega_{0}} \sum_{A \subset \mathcal{S}_{f}} \operatorname{Var}_{\mu_{\infty}^{+}}\left(P_{t} g_{\sigma, A}\right) \\
& \leqslant C_{f} \ell_{0} e^{-t^{1 / 3} / c} .
\end{aligned}
$$

Recalling (7.27), it suffices to take $\ell_{0}=t^{1 / 3}$ to conclude the proof.

We turn to the proof of the lower bound (3.12). Let $f=f^{a}, I$ be a function as in (3.11). Assume that

$$
\sqrt{t}>2 \min _{x \in I} a_{x}
$$

and let $y \in I$ be a point such that $a_{y}=\min _{x \in I} a_{x}$ (to fix ideas, we assume that $y$ is even). Let $\ell(t) \in 2 \mathbb{N}$ satisfy (7.19) for some sufficiently large $c_{1}$. We write

$$
\begin{aligned}
\operatorname{Var}_{\mu_{\infty}^{+}}\left(P_{t} f\right) & =\frac{1}{2} \mu_{\infty}^{+} \otimes \mu_{\infty}^{+}\left[\left(\left(P_{t} f\right)(\xi)-\left(P_{t} f\right)\left(\xi^{\prime}\right)\right)^{2}\right] \\
& \geqslant \frac{1}{2} \mu_{\infty}^{+} \otimes \mu_{\infty}^{+}\left[1_{\left\{\xi \in B_{y, \ell(t)}\right\}}\left(\left(P_{t} f\right)(\xi)-\left(P_{t} f\right)\left(\xi^{\prime}\right)\right)^{2}\right]
\end{aligned}
$$

where $B_{y, \ell}$ is the set defined in (7.18). As a consequence of (7.20),

$$
\operatorname{Var}_{\mu_{\infty}^{+}}\left(P_{t} f\right) \geqslant c e^{-\sqrt{t} / c} \mu_{\infty}^{+} \otimes \mu_{\infty}^{+}\left[\left(\left(P_{t} f\right)(\xi)-\left(P_{t} f\right)\left(\xi^{\prime}\right)\right)^{2} \mid \xi \in B_{y, \ell(t)}\right]
$$

By monotonicity, for every initial condition $\xi \in B_{y, \ell(t)}$ and every $s>0$ (and in particular for $s=t$ ) one has

$$
0 \leqslant\left(P_{t} f\right)(\xi) \leqslant \mathbb{E}\left[f\left(\eta^{\xi}(s)\right) \mid \eta_{y \pm \ell(t)}^{\xi}(r)=0 \forall r \leqslant s\right]
$$


From (7.23) we know that for any given $\varepsilon>0$, if $c_{1}=c_{1}(\varepsilon)$ is chosen large enough in (7.19), for every $s \leqslant t$ one has

$$
\mathbb{P}\left[\eta_{y}^{\xi}(s) \geqslant \ell(t) / 2 \mid \eta_{y \pm \ell(t)}^{\xi}(r)=0 \forall r \leqslant s\right] \geqslant 1-\varepsilon
$$

if $\xi \in B_{y, \ell(t)}$. Since $\|f\|_{\infty}=1$ and $\ell(t)>2 a_{y}$ (cf. (7.28)), this implies that if $\xi \in B_{y, \ell(t)}$, then

$$
0 \leqslant\left(P_{t} f\right)(\xi) \leqslant \varepsilon .
$$

Going back to (7.30), we obtain

$$
\operatorname{Var}_{\mu_{\infty}^{+}}\left(P_{t} f\right) \geqslant c e^{-\sqrt{t} / c}\left[\mu_{\infty}^{+}\left[\left(P_{t} f\right)^{2}\right]-2 \varepsilon\right] .
$$

Choosing $\varepsilon$ small enough and using the Cauchy-Schwarz inequality we find the estimate (3.12). Adjusting the value of $c_{f}$ yields the desired bound for all $t \geqslant 0$, i.e. without the restriction (7.28). This ends the proof of Theorem 3.6.

Acknowledgments F.L.T. would like to thank C. Bernardin, T. Bodineau, F. Caravenna and G. Giacomin for useful discussions and suggestions on the literature. P.C. thanks N. Yoshida, P. Tetali and D. Randall for useful conversations. F.L.T. was partially supported by the GIP-ANR project JC05_42461 (POLINTBIO).

\section{References}

[1] K. Alexander, The effect of disorder on polymer depinning transitions, Commun. Math. Phys., to appear. math.PR/0610008.

[2] T. Ambjörnsson, S. K. Banik, M. A. Lomholt, R. Metzler, Master equation approach to DNA breathing in heteropolymer DNA, Phys. Rev. E 75 (2007), 021908.

[3] A. Bar, Y. Kafri, D. Mukamel, Loop Dynamics in DNA Denaturation, Phys. Rev. Lett. 98 (2007), 038103.

[4] T. Bodineau, F. Martinelli, Some new results on the kinetic Ising model in a pure phase, J. Statist. Phys. 109 (2002), 207-235. MR1927919

[5] F. Caravenna, G. Giacomin and L. Zambotti, Sharp asymptotic behavior for wetting models in (1+1)-dimension, Electron. J. Probab. 11 (2006), 345-362. MR2217821

[6] B. Derrida, V. Hakim and J. Vannimenus, Effect of disorder on two-dimensional wetting, J. Statist. Phys. 66 (1992), 1189-1213. MR1156401

[7] J.-D. Deuschel, G. Giacomin and L. Zambotti, Scaling limits of equilibrium wetting models in (1+1)-dimension, Probab. Theory Rel. Fields 132 (2005), 471-500. MR2198199

[8] W. Feller, An introduction to probability theory and its applications, Vol. I, 2nd edition, John Wiley \& Sons, Inc., New York-London-Sydney, 1966. MR0210154

[9] M. E. Fisher, Walks, walls, wetting, and melting, J. Statist. Phys. 34 (1984), 667-729. MR0751710 
[10] D. Fisher and D. Huse, Dynamics of droplet fluctuation in pure and random ising systems, Phys. Rev. B, 35, 13 (1987).

[11] R. Fontes, R. Schonmann, V. Sidoravicious, Stretched exponential fixation in stochastic Ising models at zero temperature, Comm. Math. Phys. 228 (2002), 495-518. MR1918786

[12] G. Giacomin, F. L. Toninelli, Smoothing effect of quenched disorder on polymer depinning transitions, Comm. Math. Phys. 266 (2006), 1-16. MR2231963

[13] G. Giacomin and F. L. Toninelli, The localized phase of disordered copolymers with adsorption, ALEA 1 (2006), 149-180. MR2249653

[14] G. Giacomin, Random polymer models, IC Press, World Scientific (2007).

[15] T. P. Hayes, A. Sinclair, A general lower bound for mixing of single-site dynamics on graphs, Ann. Appl. Probab. 17 (2007), 931-952. MR2326236

[16] Y. Isozaki, N. Yoshida, Weakly pinned random walk on the wall: pathwise description of the phase transition, Stoch. Proc. Appl. 96 (2001), 261-284. MR1865758

[17] R. Martin, D. Randall, Disjoint decomposition of Markov chains and sampling circuits in Cayley graphs, Combin. Probab. Comput. 15 (2006), 411-448. MR2216477

[18] R. Martin, D. Randall, Sampling adsorbing staircase walks using a new Markov chain decomposition method, 41st Annual Symposium on Foundations of Computer Science, 492-502, IEEE Comput. Soc. Pres (2000) MR1931846

[19] L. Miclo, An example of application of discrete Hardy's inequalities, Markov Proc. Rel. Fields 5(3) (1999), 319-330. MR1710983

[20] Y. Peres, Mixing for Markov chains and spin systems, draft of lecture notes available at http://www.stat.berkeley.edu/ ${ }^{\sim}$ peres

[21] F. L. Toninelli, A replica-coupling approach to disordered pinning models, Commun. Math. Phys., to appear. math-ph/0701063.

[22] Y. Velenik, Localization and delocalization of random interfaces, Probability Surveys 2006, Vol. 3, 112-169. MR2216964

[23] D. B. Wilson, Mixing times of Lozenge tiling and card shuffling Markov chains, Ann. Appl. Probab. 14 (2004), 274-325. MR2023023 